\title{
Modality-Based Organization of Ascending Somatosensory Axons in the Direct Dorsal Column Pathway
}

\author{
Jingwen Niu, ${ }^{1}$ Long Ding, ${ }^{1}$ Jian J. Li, ${ }^{2}$ Hyukmin Kim, ${ }^{3}$ Jiakun Liu, ${ }^{1}$ Haipeng Li, ${ }^{1,4}$ Andrew Moberly, ${ }^{1}$ Tudor C. Badea, ${ }^{5}$ \\ Ian D. Duncan, ${ }^{6}$ Young-Jin Son, ${ }^{3}$ Steven S. Scherer, ${ }^{2}$ and Wenqin Luo ${ }^{1}$ \\ ${ }^{1}$ Department of Neuroscience and ${ }^{2}$ Department of Neurology, Perelman School of Medicine, University of Pennsylvania, Philadelphia, Pennsylvania 19104, \\ ${ }^{3}$ Shriners Hospital Pediatric Research Center and Department of Anatomy and Cell Biology, Temple University School of Medicine, Philadelphia, \\ Pennsylvania 19140, ${ }^{4}$ Department of Neurology, the First People's Hospital of Chenzhou, Chenzhou, Hunan, China, ${ }^{5}$ Retinal Circuit Development \& \\ Genetics Unit, National Eye Institute, Bethesda, Maryland 20892, and 'Department of Medical Sciences, School of Veterinary Medicine, University of \\ Wisconsin, Madison, Wisconsin 53706
}

The long-standing doctrine regarding the functional organization of the direct dorsal column (DDC) pathway is the "somatotopic map" model, which suggests that somatosensory afferents are primarily organized by receptive field instead of modality. Using modalityspecific genetic tracing, here we show that ascending mechanosensory and proprioceptive axons, two main types of the DDC afferents, are largely segregated into a medial-lateral pattern in the mouse dorsal column and medulla. In addition, we found that this modality-based organization is likely to be conserved in other mammalian species, including human. Furthermore, we identified key morphological differences between these two types of afferents, which explains how modality segregation is formed and why a rough "somatotopic map" was previously detected. Collectively, our results establish a new functional organization model for the mammalian direct dorsal column pathway and provide insight into how somatotopic and modality-based organization coexist in the central somatosensory pathway.

\section{Introduction}

The spinal cord dorsal column contains ascending axons of primary somatosensory neurons [direct dorsal column (DDC) pathway] and secondary neurons of spinal cord, and descending axons from the dorsal column nuclei (DCN). In rodents, the dorsal corticospinal tract also descends in the dorsal column. Given that the dorsal column is one of the major axonal bundles bridging the periphery and brain, it is important to thoroughly understand its normal functional organization.

The DDC is divided into a medial gracile fasciculus and a lateral cuneate fasciculus, which contains afferents of DRG neurons below and above T6, respectively, and innervate the ipsilateral DCNs of medulla (see Fig. 1A). A prevailing view on the functional organization of the DDC pathway is the "somatotopic map" model, which suggests that ascending somatosensory fibers entering at successive rostral levels are located lateral to those from lower segments (Watson and Kayalionglu, 2009). This "somatotopic map" model is supported by physiological recordings

Received Aug. 9, 2013; revised Sept. 12, 2013; accepted Sept. 29, 2013.

Author contributions: J.N., Y.-J.S., S.S.S., and W.L. designed research; J.N., J.J.L., H.K., and I.D.D. performed research; T.C.B. contributed unpublished reagents/analytic tools; J.N., L.D., J.L., H.L., A.M., and W.L. analyzed data; J.N., L.D., Y.-J.S., S.S.S., and W.L. wrote the paper.

W.L. was supported by National Institutes of Health Grants R00NS069799 and 1R01NS083702, Thomas B. MCCabe and Jeanette E. Laws McCabe Pilot Award, Basil O'Connor Starter Scholar Research Award, and the University of Pennsylvania. Y.-J.S. was supported by the National Institutes of Health and Shriners Hospitals for the Children.

The authors declare no competing financial interests.

Correspondence should be addressed to Dr. Wenqin Luo, Department of Neuroscience, Perelman School of Medicine, University of Pennsylvania, 145 Johnson Pavilion, 3610 Hamilton Walk, Philadelphia, PA 19104. E-mail: luow@mail.med.upenn.edu.

DOI:10.1523/JNEUROSCI.3429-13.2013

Copyright $\odot 2013$ the authors $\quad 0270-6474 / 13 / 3317691-19 \$ 15.00 / 0$
(Nord, 1967; Johnson et al., 1968; Whitsel et al., 1969, 1970; Culberson and Brushart, 1989), dye tracing (Maslany et al., 1991; Giuffrida and Rustioni, 1992), and lesion studies (Smith and Deacon, 1984).

On the other hand, multiple types of somatosensory afferents, two major types of which are proprioceptors and $A \beta$ lowthreshold mechanoreceptors (LTMRs), project through the DDC pathway. Because these different types of somatosensory afferents join the dorsal column from each spinal cord segment, the "somatotopic map" model predicts that they would intermingle together (see Fig. 1B). However, physiological recordings suggested that somatosensory fibers carrying the same modality of information project together in the dorsal column (Uddenberg, 1968) and innervate distinct domains of DCNs (Dykes et al., 1982; Hummelsheim et al., 1985; Fyffe et al., 1986). These seemingly contradictory observations raise the question of how the somatotopic and modality-based organization coexists in the DDC pathway.

Previously, we found that fibers of genetically traced rapidly adapting (RA) mechanoreceptors, a major type of A $\beta$ LTMR, are highly enriched in the cervical gracile fasciculus and innervate subdomains of DCNs (Luo et al., 2009). However, it is unclear whether this observation truly reflects a modality-based organization in the DDC pathway or it is simply the result of somatosensory fiber re-sorting as they ascend toward the medulla (Whitsel et al., 1970; Willis, 1991).

Using a combination of genetic and anatomical approaches, here we show that mouse mechanosensory and proprioceptive afferents are largely segregated into a medial-lateral pattern throughout the entire dorsal column. In addition, we found that 
this modality-based organization is likely to be conserved in other mammalian species, including human. Finally, using modality-specific sparse genetic tracing, we identified key morphological distinctions between mechanosensory and proprioceptive afferents, which lead to the "modality segregation" and explains why a rough "somatotopic map" was previously detected. Collectively, our results suggest that ascending somatosensory afferents in the mammalian DDC pathway are primarily organized by modality and that a somatotoptic map exists within the same modality. Our work provides a new anatomical reference for studying dorsal column development, injury, and regeneration.

\section{Materials and Methods}

Mouse strains and genetic labeling of RA mechanoreceptors and proprioceptors. Mice were raised in a barrier facility in the Hill Pavilion, University of Pennsylvania. All procedures were conducted according to animal protocols approved by the Institutional Animal Care and Use Committee of the University of Pennsylvania and the National Institutes of Health guidelines. Mice used in this paper were described previously: Ret CreERT,

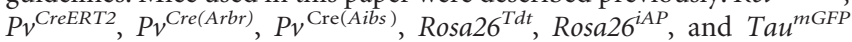
mice (Hippenmeyer et al., 2005; Badea et al., 2009; Luo et al., 2009; Madisen et al., 2010; Taniguchi et al., 2011). We set timed pregnancy mating for Ret ${ }^{\text {CreERT2 }}$ and Rosa26 $6^{\text {Tdt }}$, Tau ${ }^{\text {mGFP }}$, or Rosa $26^{\text {iAP }}$ reporter mice and treated pregnant female mice with 4-hydroxy-tamoxifen (1.5, 2, and $1 \mathrm{mg}$ at E10.5, E11.5, and E12.5) by oral gavage to specifically label the RA mechanoreceptor population. Ret ${ }^{\text {CreERT2 }}$; Rosa26 $6^{\text {iAP }}$ mice were treated with either a very low dosage of 4-hydroxy-tamoxifen (4HT; 0.05 $\mathrm{mg}$ at E12.5) or no $4 \mathrm{HT}$ (background recombination) to achieve sparse labeling of RA mechanoreceptors. As for proprioceptors, $P v^{C r e E R T 2}$ mice were mated with Rosa $26^{i A P}$ reporter line, and 20 and $10 \mathrm{mg}$ of tamoxifen was gavaged at E16.5 and 17.5, respectively, to label the population. Sparse labeling of proprioceptors was achieved by treating the $\mathrm{Pv}^{\mathrm{CreERT2}}$; Rosa26 ${ }^{i A P}$ mice with $1.5-2.5 \mathrm{mg}$ of tamoxifen at E16.5.

Tissue preparation and histology. Mice were killed with $\mathrm{CO}_{2}$, transcardially perfused with PBS $/ 4 \%$ PFA, postfixed with $4 \%$ PFA for $2 \mathrm{~h}$ at $4{ }^{\circ} \mathrm{C}$, and cryo-protected in $1 \times$ PBS, $30 \%$ sucrose overnight. Frozen sections of spinal cord and DRGs from animals younger than P21 were cut within the spinal column using a Leica CM1950 cryostat, and spinal cords of adult mice were dissected out before sectioning. Immunostaining of spinal cords, whole-mount DRGs, interosseous membrane, glabrous skin, and whole-mount hairy skin was performed as described previously (Luo et al., 2009). Antibodies and dyes used are as follows: rabbit antiparvalbumin (Swant, PV 25), goat anti-parvalbumin (Swant, PVG214), chicken anti-GFP (Aves, GFP-1020), chicken anti-NFH (Aves, NF-H), rabbit anti-NF200 (Sigma, N4142), rabbit anti-cRet (IBL, 18121), rabbit anti-CGRP (Immunostar, 24112), guinea pig anti-VGluT1 (Millipore, AB5905), rabbit anti-S100 (Dako, Z0311), and Alexa fluorescenceconjugated goat or donkey secondary antibodies (Invitrogen or Jackson ImmunoResearch Laboratories). Sections of spinal cord and wholemount DRGs of $\mathrm{Pv}^{\mathrm{CrEERT2}}$; Rosa $26^{\text {GAP }}$ mice were immunostained with parvalbumin $(\mathrm{Pv})$ antibody and then AP color reaction using HNPP-fast red substrate (Roche, 11758888001) was performed according to the manufacturer's protocol. AP color reaction with BCIP/NBT (Roche, 1138221001 and 11383213001) substrate was also performed with sections of Ret ${ }^{\text {CreERT2 }}$; Rosa26 ${ }^{\text {iAP }}$ and Pv ${ }^{\text {CreERT2 }}$; Rosa26 $6^{\text {iAP }}$ spinal cord. Whole-mount spinal cords of sparsely labeled animals were dissected out carefully with all DRGs attached, and AP color reaction with BCIP/NBT substrate was performed as previously described (Li et al., 2011).

Semithin sections of mammalian dorsal column. Mice were anesthetized with an intraperitoneal injection of xylazine and ketamine and perfused transcardially with fresh prepared fixative: $2 \%$ PFA and 2\% glutaraldehyde in in $0.1 \mathrm{~m}$ phosphate buffer, $\mathrm{pH} 7.4$, for half an hour. Deeply fixed human cervical spinal cord tissue of a 21-year old male was kindly provided by Dr. William W. Schlaepfer in the Department of Pathology at the Perelman School of Medicine, University of Pennsylvania. Fixed adult monkey spinal cord tissue was kindly provided by J. M. Wilson at the Children's Hospital of Philadelphia. Human and monkey spinal cords were dissected, cut into $2 \mathrm{~mm}$ transverse slices and postfixed in the same fixative overnight at $4^{\circ} \mathrm{C}$. Tissues were postfixed in $1 \% \mathrm{OsO}_{4}$ for $2 \mathrm{~h}$ and rinsed with $0.1 \mathrm{~m}$ phosphate buffer. After gradient ethanol dehydration and rinse in propylene oxide, tissues were embedded in Epon for cutting $1 \mu \mathrm{m}$ semithin sections. Sections were stained either in toluidine blue or paraphenylenediamene. Semithin sections of adult rat, feline, and canine cervical spinal cords were processed and sectioned by Dr. Ian D. Duncan at University of Wisconsin as previously described (Jackson et al., 2009).

Central root rhizotomy. The survival surgery of central root rhizotomy was conducted in Shriners Hospitals Pediatric Research Center, Temple University. All surgical and postoperative procedures were performed in accordance with Temple's Institutional Animal Care and Use Committee and National Institutes of Health guidelines. Dorsal root transection of L4-L5 was performed with 3 adult C57BL/6 mice (The Jackson Laboratory). Procedures are the same as previously described (Fleming et al., 2012). Two weeks after lesion surgery, animals were killed and semithin sections of the spinal cord were performed.

Heatmap generation. To visualize the distribution of dorsal column axons of different sizes, we performed the following procedures using a custom program written in MATLAB (MathWorks) (see Fig. 8A-E). (1) A raw image is converted to a binary image using a threshold that appropriately separates the cross-sections of axons and fiber walls. (2) Thresholds for the maximum cross-section area and maximum length of the major axis are operationally determined for each image to exclude most non-axonspurious areas, such as the extracellular space enclosed by the outer walls of multiple axons. (3) Axon size (in pixels) was automatically measured. (4) The cumulative percentile of all dorsal column axons in different species was plotted against axon sizes (see Fig. $8 G$ ), and a value corresponding to a specific percentile was determined as the threshold for large/small categories in a given species (see Fig. $8 F$ ). We used $88 \%$ as the threshold for mouse and rat and $80 \%$ as the threshold for feline, canine, monkey, and human. (5) Densities for all axons, large axons only, and small axons only were computed using a sliding window. An axon is counted if its centroid lies within the sliding window. (6) Axon density data are plotted in a heatmap, with warm/cool colors corresponding to high/low density and mapped to the full range of values for each image and axon type.

Our selection of category boundary for large/small axons was based on axon size distributions of identified mouse RA mechanoreceptors and proprioceptors. Figure $8 F$ plots the probability density functions for the two axon types. With a threshold of $4.8 \mu \mathrm{m}^{2}, 95 \%$ of mouse proprioceptive axons (green trace) were included in the "large" category, whereas $90.8 \%$ of mouse RA mechanosensory afferents (red trace) were included in the "small" category. With a threshold of $3.08 \mu \mathrm{m}^{2}, 99 \%$ of mouse proprioceptors were included in the "large" category, whereas $81.1 \%$ of mouse RA mechanosensory afferents were included in the "small" category. Because the axon sizes differ among species (see Fig. $8 G, H$ ), we opted to use threshold values based on a common cumulative percentile of all dorsal column axons, instead of a common absolute value, for different species. In mouse, the thresholds of 3.08 and $4.8 \mu \mathrm{m}^{2}$ correspond to 80 th and 88 th cumulative percentile (black trace), respectively. Thus, we chose 88 th percentile as the category boundary for mouse and rat, which have sharper early rise in their cumulative functions, and 80th percentile for feline, canine, monkey, and human, which have slower rise in their cumulative functions (see Fig. 8G,H).

Image acquisition and $3 D$ reconstruction. Fluorescent images were acquired on a Leica SP5II confocal microscope. Medulla sections with triple immunostaining were imaged using the tiling mode and stitched automatically. Large specimens, including whole-mount spinal cord of sparsely labeled animals and semithin sections of different mammalian species, were imaged on Leica DM5000B microscope with a motor stage and power-mosaic mode. Whole-mount spinal cord images were taken using a $10 \times$ objective of NA 0.40 , and semithin sections were images with a $40 \times$ objective of NA 1.25. The ascending and descending information of all labeled cells was summarized and represented in bar graphs using a program written in MATLAB (MathWorks). Cell bodies of single DRG neurons and dorsal view images of spinal cord were acquired with a Leica DFC 295 color camera. For reconstruction, image tiles of central projection of sparsely labeled neurons, including both ascending and descend- 


\section{A}

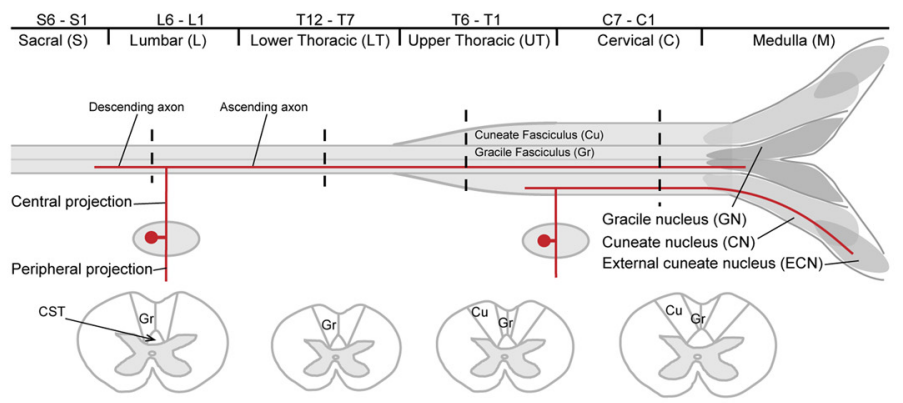

B

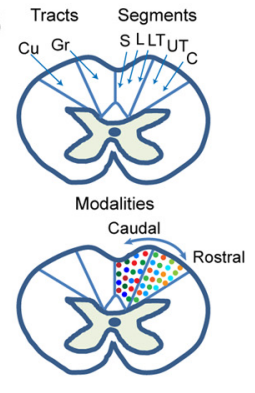

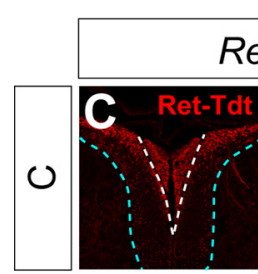
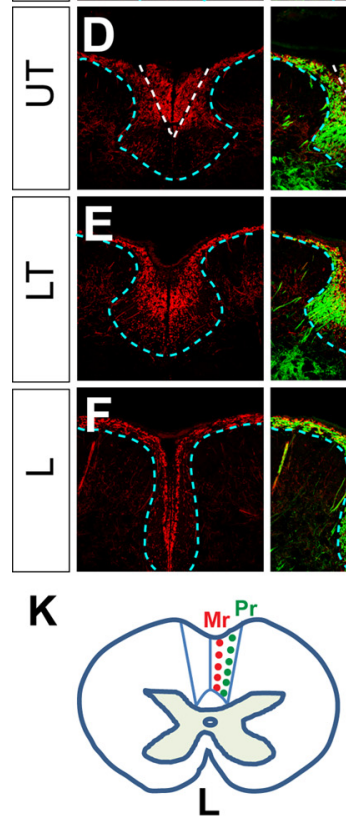

Pr: Proprioceptor

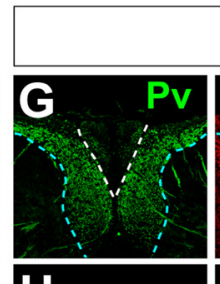

Wild Type
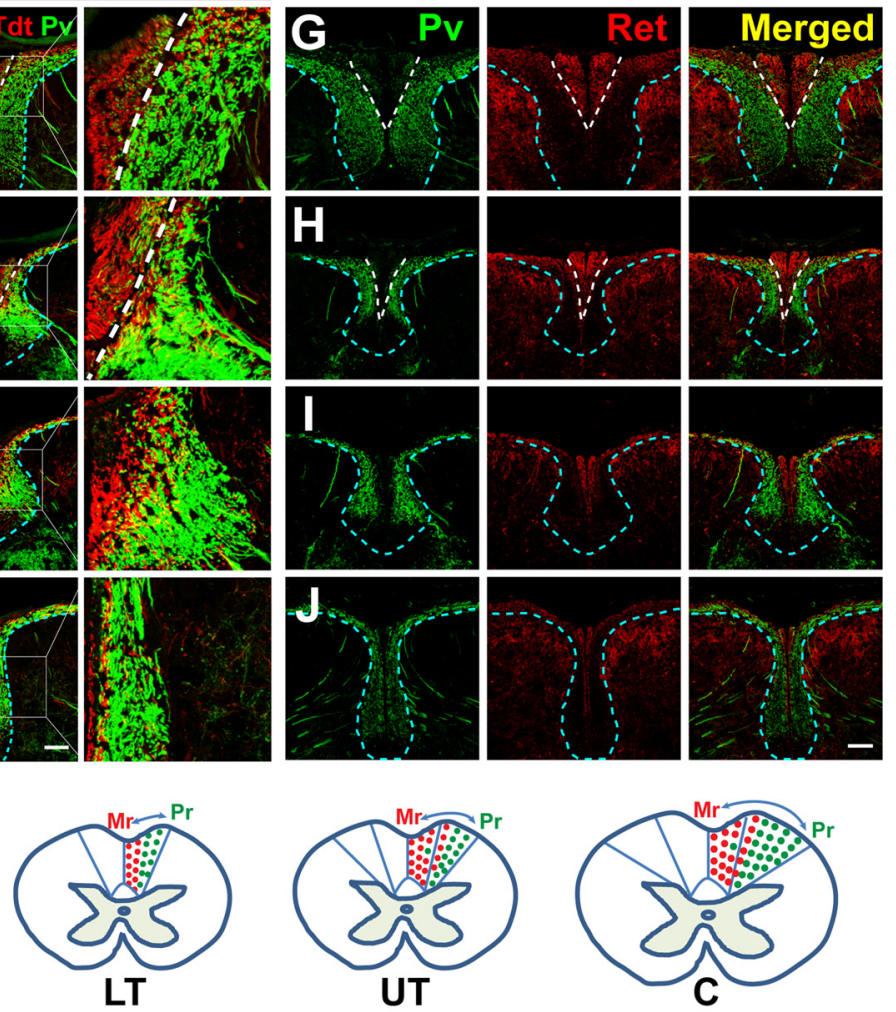

\section{Mr: Mechanoreceptor}

Figure 1. The DDC pathway and modality segregation of ascending axons of RA mechanoreceptors and proprioceptors in the P7 mouse dorsal column. $A$, Schematic illustration shows the rodent dorsal column in both longitudinal and cross section views. The dorsal column is composed of three tracts: (1) the medial gracile fasciculus (Gr) through the entire dorsal column; (2) the lateral cuneate fasciculus (Cu) that formed above thoracic segment 6 (T6), which innervate the $\mathrm{GN}, \mathrm{CN}$, and ECN in the medulla; and (3) the corticospinal tract (CST) at the most ventral area of the dorsal column. Primary somatosensory afferents from S6-T7 and T6-C1 DRGs travel in the Gr and Cu, respectively. S, Sacral; L, lumbar; LT, lower thoracic; UT, upper thoracic; C, cervical. B, Illustration of the "somatotopic map" model for the cervical mammalian dorsal column. Different colors represent different types of somatosensory afferents, and the dark to light gradient of color represents caudal to rostral segments. C-F, Immunostaining of P7 Ret ${ }^{\text {CreERT2; }}$ Rosa2 $6^{\text {Tdt }}$ spinal cord sections with Pv antibody. RA mechanoreceptors are specifically labeled by Tdt, and proprioceptors are stained by Pv. Ascending axons of mechanoreceptors and proprioceptors are segregated from each other in a medial-lateral pattern at different levels of the spinal cord. G-J, Immunostaining of Pv and Ret antibodies of P7 WT mouse spinal cord sections. Ret antibody stains RA mechanosensory axons in the dorsal column, whereas Pv antibody stains proprioceptive axons. Ascending axons of RA mechanoreceptors and proprioceptors are segregated from each other in a medial-lateral pattern at different levels of the spinal cord. $n=3$ mice. Scale bar, $50 \mu \mathrm{m}$. $\boldsymbol{K}$, Illustration of spinal cord transverse views to depict the modality-based segregation of ascending axons of RA mechanoreceptor (Mr) (red) and proprioceptors (Pr) axons (green) at L, LT, UT, and C levels.

ing fibers with collaterals and arbors, were taken using the greyscale format of the camera in a $z$-stack manner with $\sim 100-200$ steps and step size of $1 \mu \mathrm{m}$, using a $20 \times$ objective of NA 0.70 . A total of $16-25$ tiles for each cell were taken because the central projections of RA mechanoreceptors and proprioceptors are $\sim 1-6 \mathrm{~cm}$ long. Image alignment and 3D reconstruction were performed in Neuromantic (Darren Myat, available at http://www.reading.ac.uk/neuromantic), an open-source freeware widely used in neuronal tracing. Reconstructions were exported to the Rotator visualization software using scripts written in MATLAB (Badea and Nathans, 2004; Shi, 2013).

Quantification and statistics. Cell number counting and measurement of transverse areas of dorsal column axons were performed using ImageJ.
Column graphs and scatter plots were generated in GraphPad Prism 5. All error bars are \pm SEM. Student's $t$ test or one-way ANOVA was used to compare the significances between different groups.

\section{Results}

Modality segregation in the P7 mouse dorsal column and DCNs

To determine whether the DDC pathway is primarily organized by modality, we generated $\operatorname{Ret}^{\text {CreERT2 }}$; Rosa $26^{T d t}$ mice, in which RA mechanoreceptors are specifically labeled with a red fluorescent protein, Tdtomato (Tdt) (Luo et al., 2009). We stained P7 


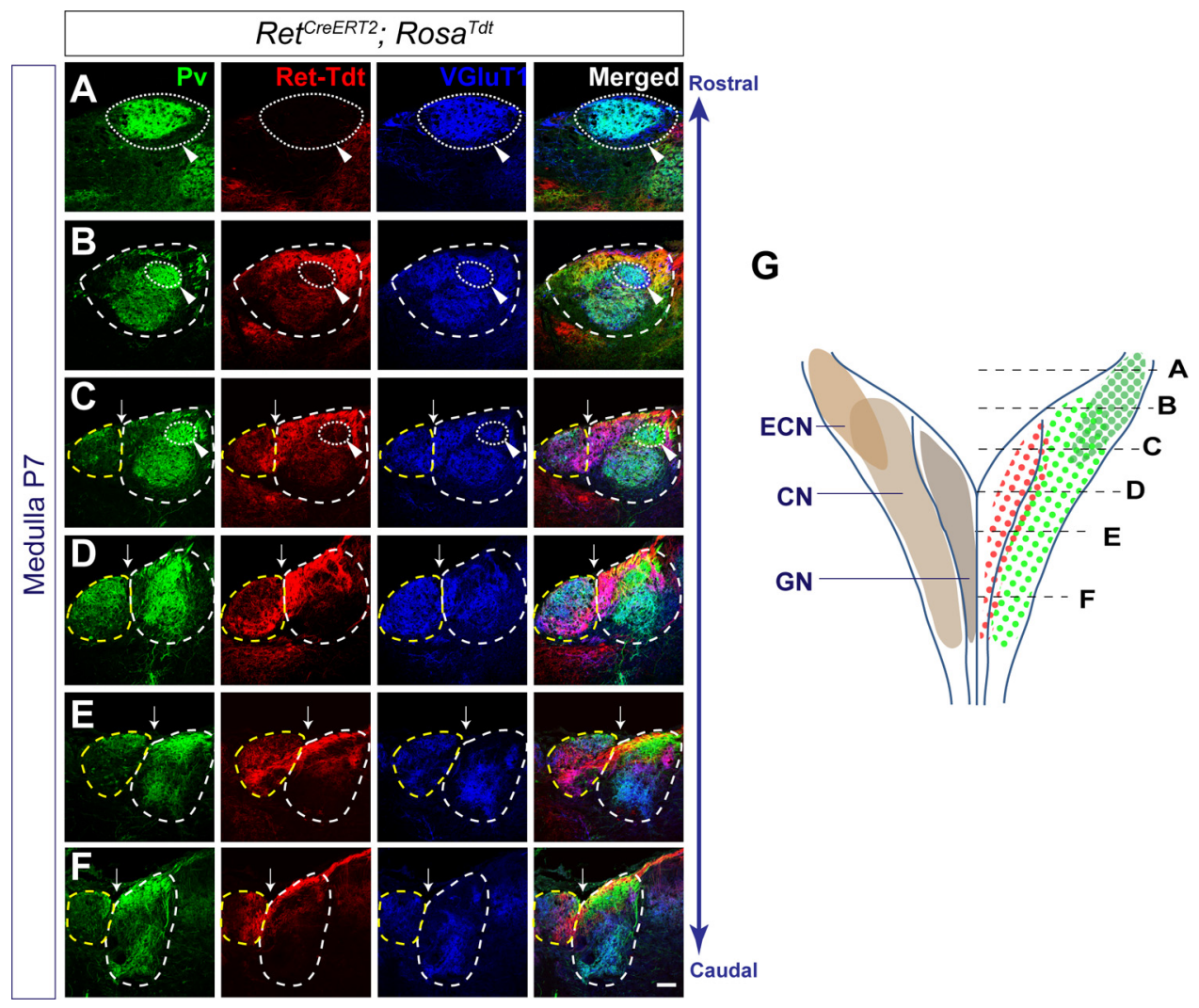

Figure 2. Modality segregation of RA mechanoreceptors and proprioceptors in the P7 mouse DCNs. A-F, Representative images from serial sections of a P7 Ret ${ }^{\text {CreERT2 }}$; Rosa $26^{\text {Tdt }}$ mouse medulla, which were stained with antibodies against Pv and VGluT1. VGluT1 stains synapses of primary mechanosensory and proprioceptive afferents and thus was used as a marker together with the dorsal sulcus (white arrows) to define the DCNs. Dashed yellow circle represents GN; dashed white circle represents CN; dotted white circle and white arrowheads represent ECN. At caudal levels, Tdt ${ }^{+}$RA mechanoreceptors mainly innervate the lateral part of $\mathrm{GN}$, whereas proprioceptors innervate $\mathrm{CN}(\boldsymbol{E}, \boldsymbol{F})$. At more rostral levels, RA mechanoreceptors start to innervate the dorsal medial part of $\mathrm{CN}_{\text {, }}$ but their termination domain is still segregated from that of proprioceptors $(\boldsymbol{C}, \boldsymbol{D})$. At the most rostral levels where $\mathrm{GN}$ ends, proprioceptors highly innervate the ECN $(\boldsymbol{A}, \boldsymbol{B})$. There are some Pv ${ }^{+}$ interneurons present in GN and CN. Scale bar, $100 \mu \mathrm{m}$. G, Schematic view of the DCNs shows the section levels and innervation pattern of RA mechanoreceptors (red dots) and proprioceptors (light and dark green dots indicate innervation domains in $\mathrm{CN}$ and ECN, respectively).

$\operatorname{Ret}^{\text {CreERT2}}$; Rosa26 ${ }^{\text {Tdt }}$ spinal cord sections with an antibody against parvalbumin $(\mathrm{Pv})$, a proprioceptor-specific marker at this developmental stage (Ernfors et al., 1994). Strikingly, in the lumbar and lower thoracic dorsal column, which only contains the gracile fasciculus, $\mathrm{Tdt}^{+}$fibers are enriched in the medial part of the gracile fasciculus, whereas $\mathrm{Pv}^{+}$fibers are located more laterally (Fig. $1 E, F$ ); in the upper thoracic and cervical dorsal column, which contains both the gracile and cuneate fasciculi, $\mathrm{Tdt}^{+}$fibers are highly enriched in the gracile fasciculus and a thin medial zone of the cuneate fasciculus, whereas $\mathrm{Pv}^{+}$fibers are located in the lateral cuneate fasciculus (Fig. 1C,D). These results suggest that RA mechanosensory and proprioceptive afferents form a complementary pattern throughout the entire dorsal column. Because Tdt and Pv label mechanoreceptors and proprioceptors in both caudal and rostral DRGs, this medial-lateral segregation pattern cannot be explained by the "somatotopic map" model. Instead, our results argue that ascending axons of mechanoreceptors and proprioceptors are largely segregated by modality in the dorsal column (Fig. $1 K$ ). Consistently, we found a similar segregation pattern when we stained P7 wild-type (WT) mouse spinal cord sections with antibodies against Ret and Pv (Fig. 1G-J). Furthermore, we examined innervation patterns of mechanoreceptors and proprioceptors in DCNs by staining serial sections of P7 Ret ${ }^{\text {CreERT2 }}$; Rosa $26^{\text {Tdt }}$ medulla with antibodies against Pv and VGluT1, which marks the DCN nucleus. We found that RA mechanoreceptors and proprioceptors innervate complementary DCN regions (Fig. 2): mechanoreceptors mainly innervate the gracile nucleus (GN) and a dorsal medial domain of the cuneate nucleus $(\mathrm{CN})$ whereas proprioceptors innervate the external cuneate nucleus (ECN) and the ventral lateral domain of $\mathrm{CN}$. This complementary innervation pattern in the DCNs further supports modality-based organization in the DDC pathway.

\section{Dynamic expression of $P v$ in mechanoreceptors and proprioceptors during development}

Since previous studies on dorsal column organization were conducted using adult animals (Whitsel et al., 1969; Maslany et al., 1991), we asked whether this modality-based functional organization is preserved in adult mice. We turned to genetic tracing for labeling adult proprioceptors as $P v$ is greatly downregulated after postnatal day 14 (P14). We first crossed $P v^{\operatorname{Cre}(A r b r)}$ knockin mice with a Tau $^{\text {mGFP }}$ reporter line (Hippenmeyer et al., 2005) in which the myristoylated green fluorescent protein (mGFP) is expressed in neurons upon Cre activation to permanently label proprioceptors. Surprisingly, we observed a number of limb-level GFP ${ }^{+}$ DRG neurons are $\mathrm{Pv}^{-}$at P7 (Fig. $3 A-C$ ). In addition, GFP ${ }^{+}$ fibers innervate not only anticipated proprioceptor target regions, such as Clarke's column and the ventral horn motor neurons, but also layers III-V of the dorsal spinal cord (Fig. $3 D, E)$. These results suggest that some mechanoreceptors are also labeled by this $P v^{\mathrm{Cre}(\mathrm{Arbr})}$ line. Indeed, $\mathrm{GFP}^{+}$fibers innervate mechanosensory end organs in the periphery, including Messeiner corpuscles, Pacinian corpuscles, and Lanceolate endings (Fig. $3 F-H$ ). A similar observation was recently reported by an- 


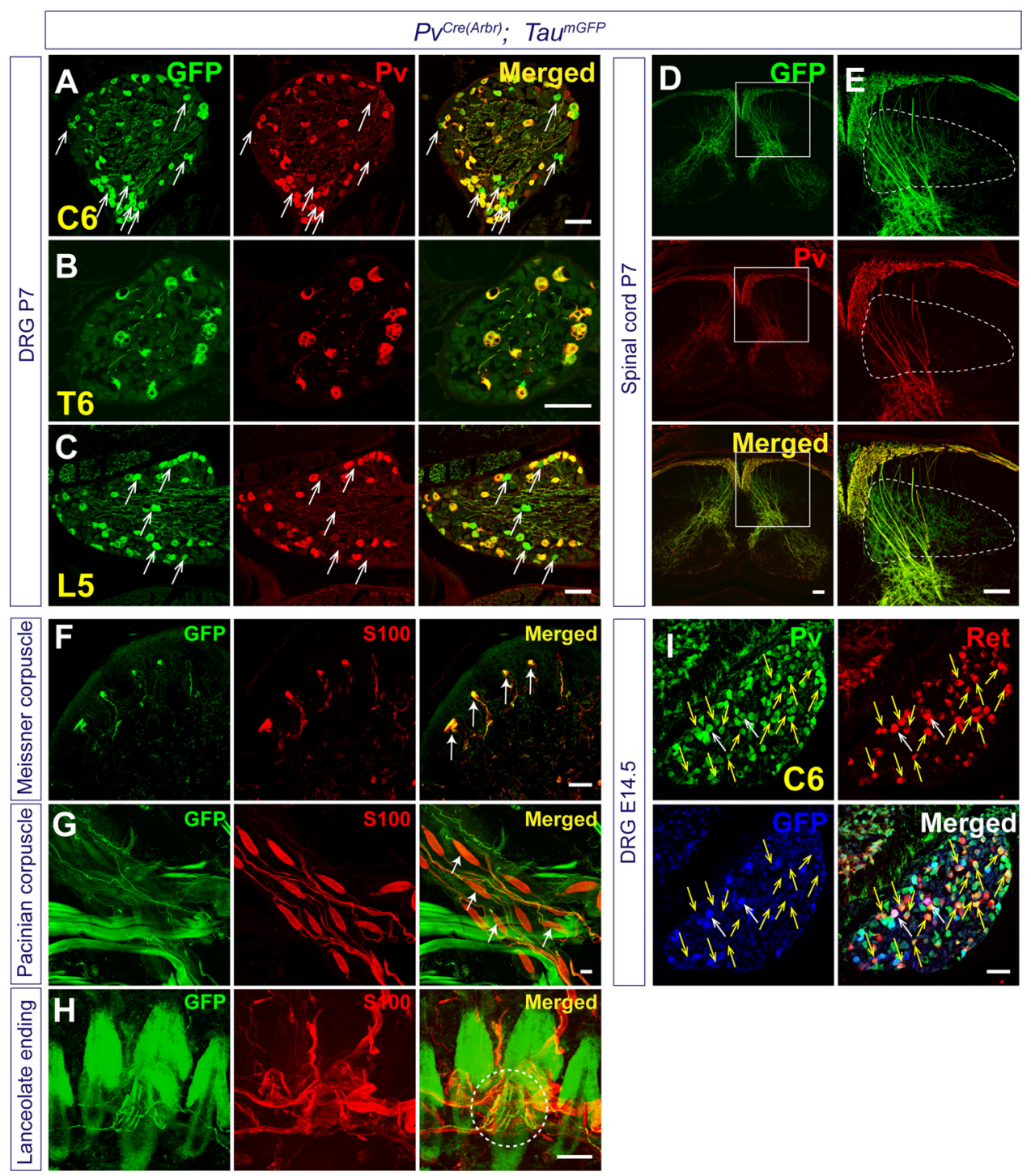

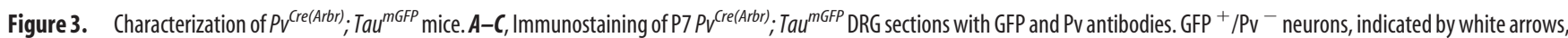
were found in the limb-level DRGs, such as C6 ( $\boldsymbol{A})$ and L5 (C), but not in non-limb-level DRGs, such as T6 (B). Scale bar, $100 \mu \mathrm{m}$. D, Low magnification of a P7 Pv ${ }^{\text {(re( }(A r b r)}$; Tau ${ }^{\text {mGFP }}$ spinal cord, double-stained with antibodies against GFP and Pv. GFP ${ }^{+} / \mathrm{Pv}^{+}$fibers innervate the intermediate Clarke's nucleus and ventral horn of the spinal cord. $\boldsymbol{E}$, High magnification of double-stained $P V^{\text {Cre(Arr) }^{2} \text {; Tau }}{ }^{\text {mGFP }}$ dorsal spinal cord shows boxed area in $\mathbf{D}$. GFP ${ }^{+}$fibers also innervate layer III to IV of the dorsal horn, circled with dashed white lines, which are Pv ${ }^{-}$. This staining pattern is consistent with previous report that Pv is a proprioceptor-specific marker at P7. $\boldsymbol{F}-\boldsymbol{H}$, Double staining of GFP and S100 in P21 PV ${ }^{\text {(ree(Arbr) }}$; Tau $^{\text {mGFP }}$ mice showing that GFP ${ }^{+}$fibers innervate mechanosensory end organs in the periphery, including Meissner corpuscles (white arrow) in the dermal papillae of hindpaw footpads $(\boldsymbol{F})$, Pacinian corpuscles (white arrow) in the interosseous membrane of fibula (G), and longitudinal lanceolate endings (circled by a dashed white ellipse) associated with follicles on back hairy skin (H). $\boldsymbol{I}$, Triple staining of PV, Ret, and GFP of E14.5PV (ree(Arbr); $\mathrm{Tau}^{\mathrm{mGFP}}$ DRG sections shows that Cre-mediated recombination begins at this stage, as some $\mathrm{Pv}^{+}$neurons have now initiated the expression of GFP. Yellow arrows indicate Pv ${ }^{+} / \mathrm{Ret}^{+}$cells, and white arrows indicate $\mathrm{GFP}^{+} / \mathrm{Pv}^{+} / \mathrm{Ret}^{+}$neurons. Scale bar, $50 \mu \mathrm{m} . n=3$ for each age.

other group (de Nooij et al., 2013). To determine when GFP starts to be expressed in mechanoreceptors, we stained E14.5 $P v^{\text {Cre(Arbr) }}$; Tau ${ }^{\text {mGFP }}$ DRG sections with GFP, Pv, and Ret antibodies. Interestingly, we found that some limb-level $\mathrm{Pv}^{+} / \mathrm{GFP}^{+}$ DRG neurons are $\operatorname{Ret}^{+}$(Fig. $3 I$ ), suggesting that $\mathrm{Pv}$ is normally expressed in some mechanoreceptors during development.

To determine the dynamic expression of $P v$ in RA mechanoreceptors during development, we examined the overlapping expression of Pv and Ret in all DRGs at E14.5, P0, and P7 (Fig. 4). We found that $\mathrm{Pv}^{+} / \mathrm{Ret}^{+}$neurons are mainly observed at limb levels at E14.5 and P0 (Fig. 4A), whereas the percentage of $\mathrm{Pv}^{+} /$ Ret ${ }^{+}$neurons over total $\mathrm{Pv}^{+}$neurons decreases postnatally (Fig. $4 B)$. Very few Ret $^{+}$DRG neurons retain expression of Pv by P7, consistent with the notion that $\mathrm{Pv}$ is a specific marker for proprioceptors at this stage (Ernfors et al., 1994). Together, our data reveal that $P v$ is transiently expressed in some limb-level mechanoreceptors during embryonic development but becomes specific for proprioceptors postnatally.

\section{Modality segregation in the adult mouse dorsal column} and DCNs

To specifically label proprioceptors in adult mice, we used $P v^{C r e E R T 2}$ mice in which the inducible Cre was knocked into the $P v$ locus (Taniguchi et al., 2011). Somehow the recombination efficiency of this $P v^{C r e E R T 2}$ line is extremely low. Given the low percentage of $\mathrm{PV}^{+}$mechanoreceptors and the decreasing expression of $P v$ in neonatal mechanoreceptors, we reasoned that the probability of labeling $\mathrm{Pv}^{+}$mechanoreceptors would be almost negligible if we treat the $P v^{C r E E R T 2}$ mice with tamoxifen at a late embryonic stage. To test this idea, we crossed $P v^{C r e E R T 2}$ mice to a 
A
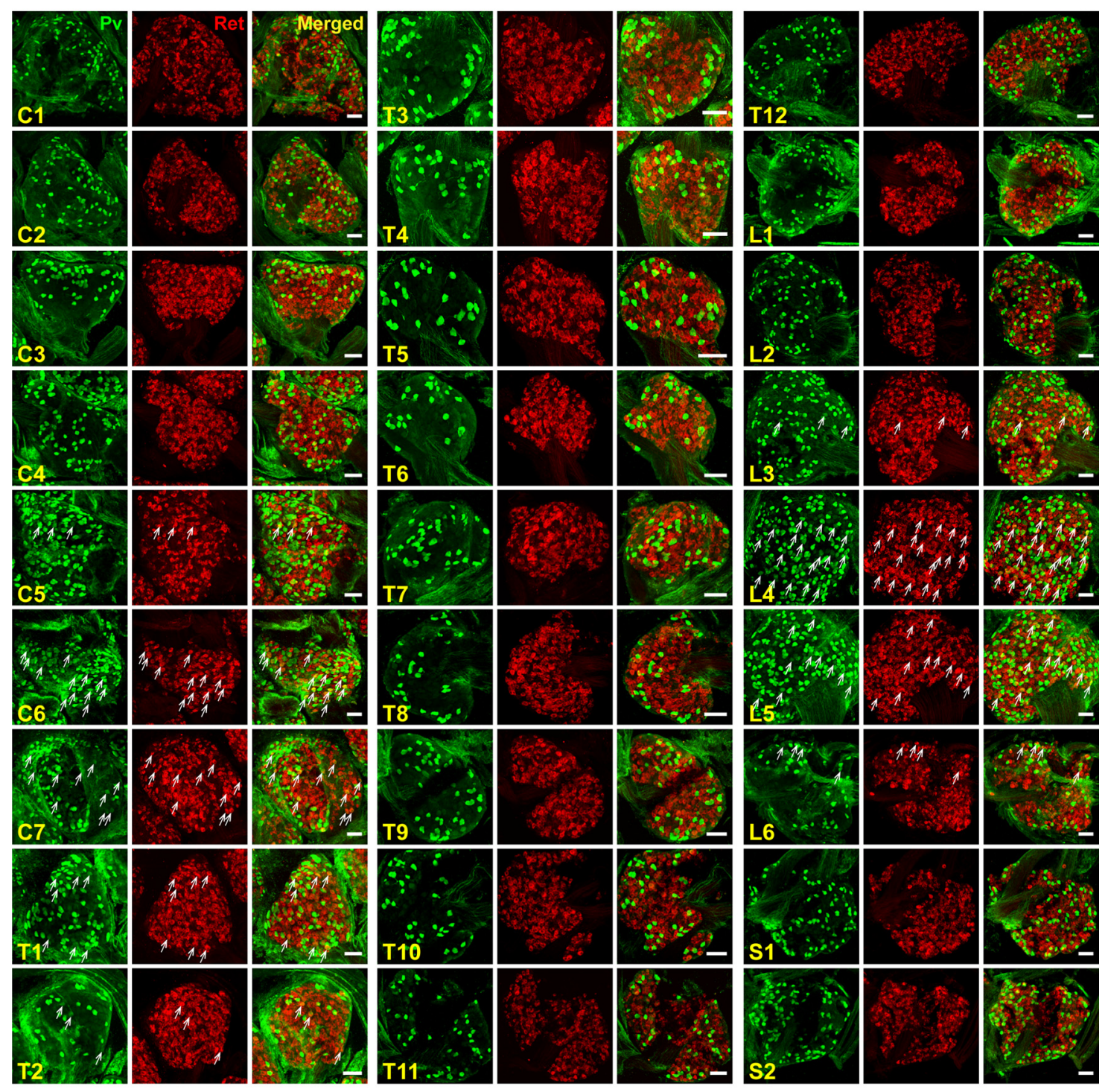

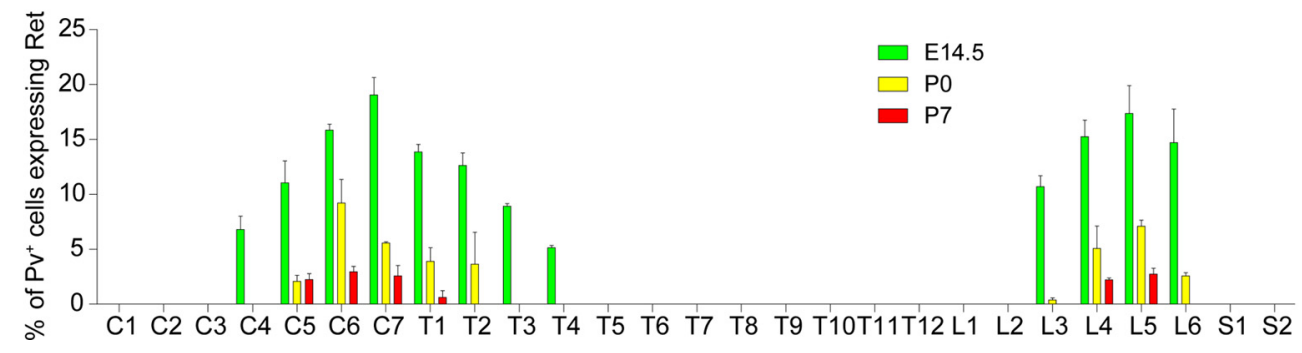

Figure 4. Dynamic expression of Pv in Ret ${ }^{+}$neurons during development. $A$, Whole-mount staining of Pv and Ret of PO WT DRGs shows the expression of Pv in Ret ${ }^{+}$DRG neurons at limb levels.

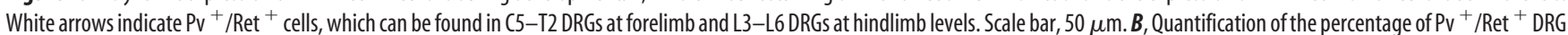
neurons over the total number of $\mathrm{Pv}^{+}$neurons in different DRGs at E14.5, P0, and P7. Error bars indicate SEM. $n=4$ mice for each developmental stage. The expression of Pv is mainly found in limb-level Ret ${ }^{+}$DRG neurons, which decreases postnatally.

Rosa26 ${ }^{i A P}$ reporter line in which human placental alkaline phosphatase (AP) is expressed upon Cre activation (Badea et al., 2009) and treated pregnant female mice with tamoxifen at E16.5 and E17.5. We found that $97.6 \%$ of the $\mathrm{AP}^{+} \mathrm{DRG}$ neurons are $\mathrm{Pv}^{+}$at $\mathrm{P} 7$ (Fig. $5 A, B$ ). In addition, $\mathrm{AP}^{+}$somatosensory fibers innervate the Clarke's nucleus and the ventral horn, but not the dorsal spinal cord (Fig. 5C-J), indicating that proprioceptors are indeed specifically labeled using this strategy.
To determine whether "modality segregation" is maintained in adult mice, we examined adult $P v^{\text {CreERT2 }}$; Rosa $26^{i A P}$ mice in which proprioceptors are genetically labeled by AP and Ret $^{\text {CreERT2 }}$; Tau ${ }^{\text {mGFP }}$ mice in which mechanoreceptors are genetically labeled by myristoylated GFP (Luo et al., 2009). Consistent with the $\mathrm{P} 7$ results, we found that $\mathrm{AP}^{+}$proprioceptive fibers show a complementary pattern to that of $\mathrm{GFP}^{+}$mechanoreceptors in the adult mouse dorsal column at different spinal cord 

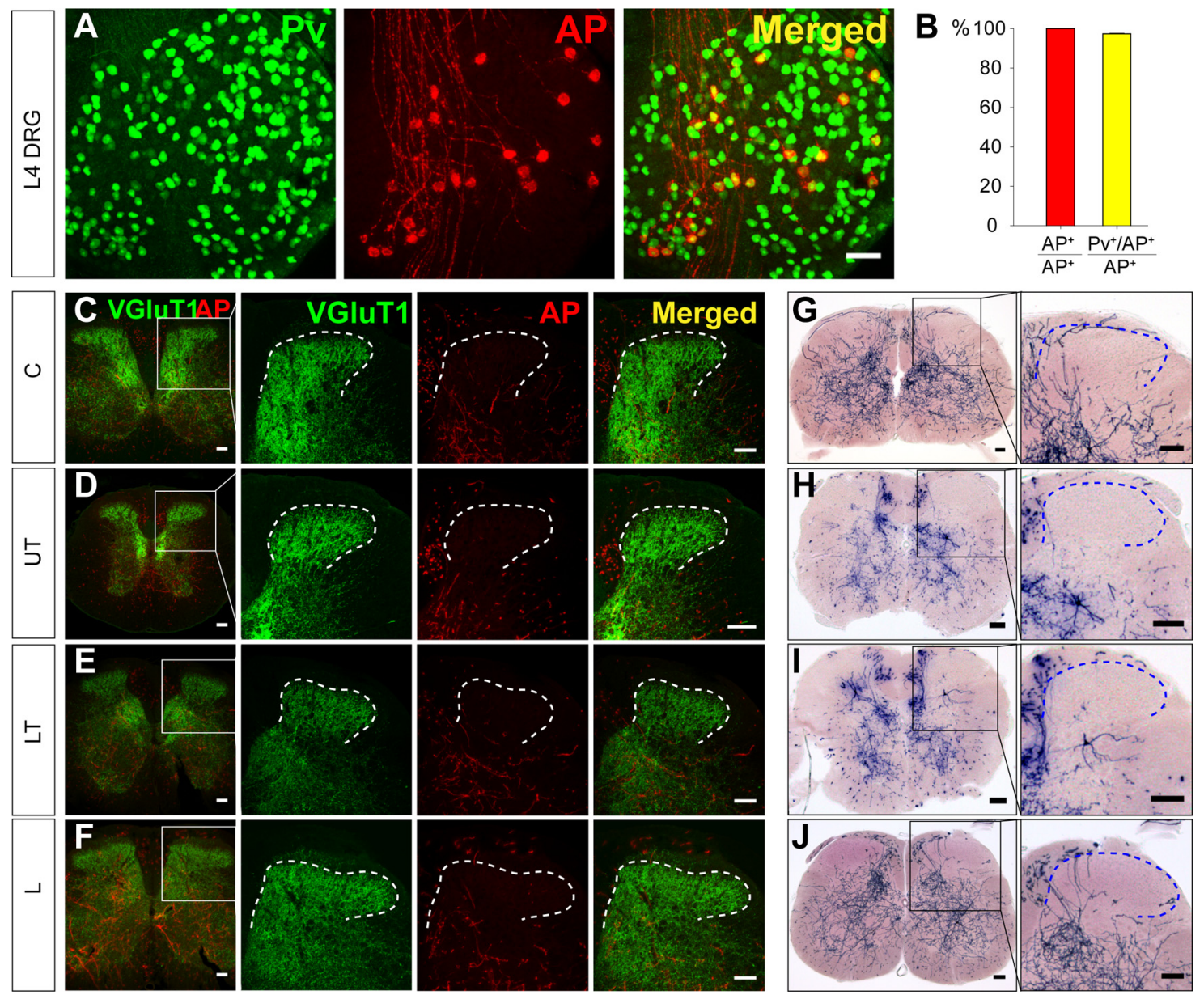

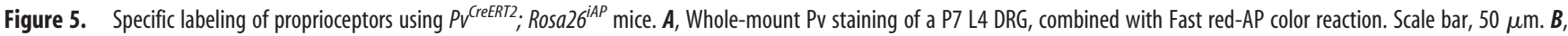
Quantification of $\mathrm{AP}^{+}$and $\mathrm{Pv}^{+} / \mathrm{AP}^{+} \mathrm{DRG}$ neurons over the total number of $\mathrm{AP}^{+}{ }^{+} \mathrm{DRG}$ neurons in $P v^{\text {CreERT2}}$; Rosa26 $6^{\text {iAP }}$ mice $(n=4) .97 .6 \%$ of all AP ${ }^{+}$neurons are Pv ${ }^{+}$proprioceptors at P7. $\boldsymbol{C}-\boldsymbol{F}$, Fastred-AP color reaction and VGluT1 staining of adult $P V^{\text {CreERT2; }}$ Rosa $26^{\text {iAP }}$ spinal cord sections at cervical $(\boldsymbol{C})$, upper thoracic $(\boldsymbol{D})$, lower thoracic $(\boldsymbol{E})$, and lumbar levels $(\boldsymbol{F})$. $A P^{+}$fibers do not innervate the dorsal horn (dashed white lines), indicating that mechanoreceptors are not labeled in Pv ${ }^{\text {CreeRT2 } ; ~ R o s a 2 ~} 6^{\text {iAP }}$ mice. Scale bar, $100 \mu \mathrm{m} . \mathbf{G}-J$, BCIP/NBT AP color reaction of the PV ${ }^{\text {CreERT2 }}$; Rosa2 ${ }^{\text {iAP }}$ spinal cord sections revealed the specific innervation pattern of proprioceptors at different levels. Dashed blue lines indicate the dorsal horn. Few Pv ${ }^{+}$spinal cord interneurons are also labeled. Scale bar, $100 \mu \mathrm{m} . n=3$.

segments (Fig. $6 A-H$ ), indicating that this modality segregation is maintained in adult. In addition, axons of mechanoreceptors and proprioceptors innervate distinct domains in the adult mouse medulla (Fig. 6I-R), a pattern similar to the one observed at P7. Remarkably, the innervation pattern of mechanoreceptors and proprioceptors in mouse DCNs revealed by our genetic tracing is in agreement with what has been suggested by physiological recordings in other mammalian species (Dykes et al., 1982; Hummelsheim et al., 1985; Fyffe et al., 1986). Collectively, our results suggest that ascending axons of mechanoreceptors and proprioceptors preferentially travel in the medial and lateral mouse dorsal column, respectively, and correspondingly innervate distinct DCN domains.

Modality segregation revealed by distribution of small- and large-diameter axons in the mouse dorsal column

To further test whether the modality-based segregation pattern is present in other mammalian species (in which genetic tracing is not feasible), we used the transverse area of dorsal column fibers as a surrogate identifier of modality. We first validated this method in mice. We genetically labeled mechanoreceptors and proprioceptors with AP using $\operatorname{Ret}^{\mathrm{CrEERT2}}$; Rosa26 $6^{i A P}$ and $P v^{C^{C r E E R T 2}}$; Rosa26 $6^{i A P}$ mice (Fig. $\left.7 A-D\right)$, respectively, and quanti- fied transverse areas of $2840 \mathrm{AP}^{+}$mechansoensory axons and 150 $\mathrm{AP}^{+}$proprioceptive axons using semithin sections $(1 \mu \mathrm{m})$ (Fig. $7 E)$. We found that most $\mathrm{AP}^{+} \mathrm{RA}$ mechanosensory axons are small-diameter myelinated axons $\left(90.8 \%<4.8 \mu \mathrm{m}^{2}\right.$, Figs. $7 B$, red arrows, $E, F, G$ and $8 F$, black arrow), whereas very few of them are of large diameter in Ret $^{\text {CreERT2 }}$; Rosa26 ${ }^{\text {iAP }}$ dorsal column (Fig. $7 B, E$, magenta arrows, $G$, inlet). To address whether these largediameter $\mathrm{AP}^{+}$axons are mechanosensory axons as well, we conducted whole-mount AP color reaction of spinal cord sections at different levels. We found that $\mathrm{AP}^{+}$somatosensory fibers highly innervate layers III-V of the $\operatorname{Ret}^{\mathrm{CrEERT2}}$; Rosa26 ${ }^{i A P}$ spinal cord (Fig. $7 J$ ) but rarely display the characteristic proprioceptive innervation pattern. Thus, this minor population of large-diameter $\mathrm{AP}^{+}$axons is likely to be mechanosensory axons, probably axons of Pacinian corpuscle neurons. Nevertheless, we could not rule out the possibility that few proprioceptors were labeled in $\operatorname{Ret}^{\mathrm{CreERT2}}$; Rosa $26^{i A P}$ mice. Almost all $\mathrm{AP}^{+}$proprioceptive axons are largediameter myelinated axons $\left(95 \%>4.8 \mu \mathrm{m}^{2}\right.$, Figs. $7 D$, green arrows, $E, H, I$, and $8 F$, black arrow).

Interestingly, on semithin sections of adult WT mouse cervical spinal cord, small-diameter axons are enriched in the gracile fasciculus and a medial marginal zone of the cuneate fasciculus, whereas large-diameter axons are enriched in the lateral part of 

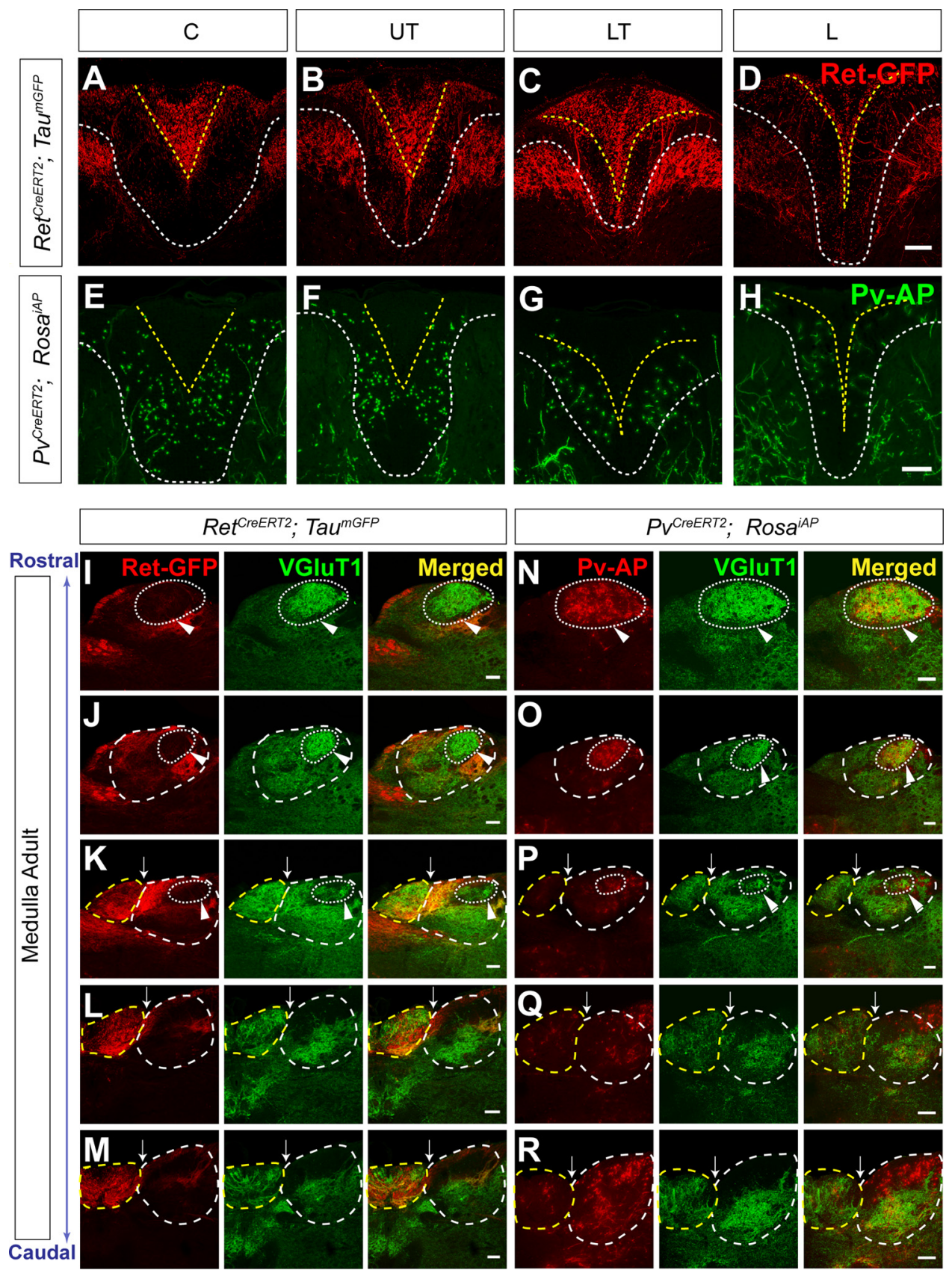

Figure 6. Complementary innervation pattern of RA mechanoreceptors and proprioceptors in the adult mouse dorsal column and DCNs. A-D, Immunostaining of adult Ret ${ }^{\text {CreeRT2. Tau }}{ }^{\text {mGFP }}$ spinal cord sections with GFP antibody. GFP ${ }^{+}$ascending axons of RA mechanoreceptors are enriched in the middle of the adult dorsal column. $\mathbf{E}-\boldsymbol{H}$, Fluorescent AP color reaction of adult $P v^{\text {creeRT2. }}$

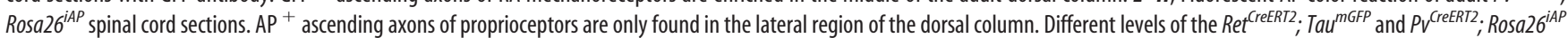
spinal cord are matched and compared. Dashed yellow lines indicate the segregation between $\mathrm{AP}^{+}$proprioceptive axons and GFP ${ }^{+}$RA mechanosensory axons, and dashed white lines indicate the boundary between the dorsal column and the dorsal horn. Scale bar, $100 \mu \mathrm{m}$. I-M, Adult medulla innervation pattern of RA mechanoreceptors, which were genetically labeled by GFP in Ret ${ }^{\text {CreeRT2; }}$ Tau $^{\text {mGFP }}$ mice. $\boldsymbol{N}-\boldsymbol{R}$, Adult medulla innervation pattern of proprioceptors, which were genetically labeled by AP in $P_{V}^{\text {CreeRT2 }}$; Rosa $26^{\text {iAP }}$ mice. Serial sections were cut through the entire medulla oblongata and costained with VGluT1. Representative images from different levels (similar to those of P7) are shown. RA mechanoreceptors and proprioceptors innervate distinct domains of DCNs, showing a complementary pattern. Dashed yellow lines, dashed white lines, and dotted white lines circle GN, CN, and ECN, respectively, as defined by VGluT1 staining and the dorsal sulcus (white arrows). The ECN is also indicated by white arrowheads. Scale bar, $100 \mu \mathrm{m} . n=3$ for both genotypes.

the cuneate fasciculus (Fig. $9 A-D$ ). On lumbar semithin sections, small-diameter axons are highly enriched in the middle of the gracile fasciculus, whereas large-diameter axons are mainly found in the lateral part of the gracile fasciculus (Fig. $9 E-G$ ). Density heatmaps that reflect the distribution of small- and largediameter axons (Fig. 8A) show a medial-lateral distinction (Fig. $9 H-K)$ on both cervical and lumbar spinal cord sections, which fits really well with the genetic tracing pattern (Figs. 1 and 6).
These results suggest that we could coarsely map the distribution of mechanosensory and proprioceptive axons based on their axon sizes.

\section{Modality segregation in the dorsal column is conserved} in mammals

Is this modality-based functional organization of the mouse dorsal column conserved in other mammalian species? To answer 

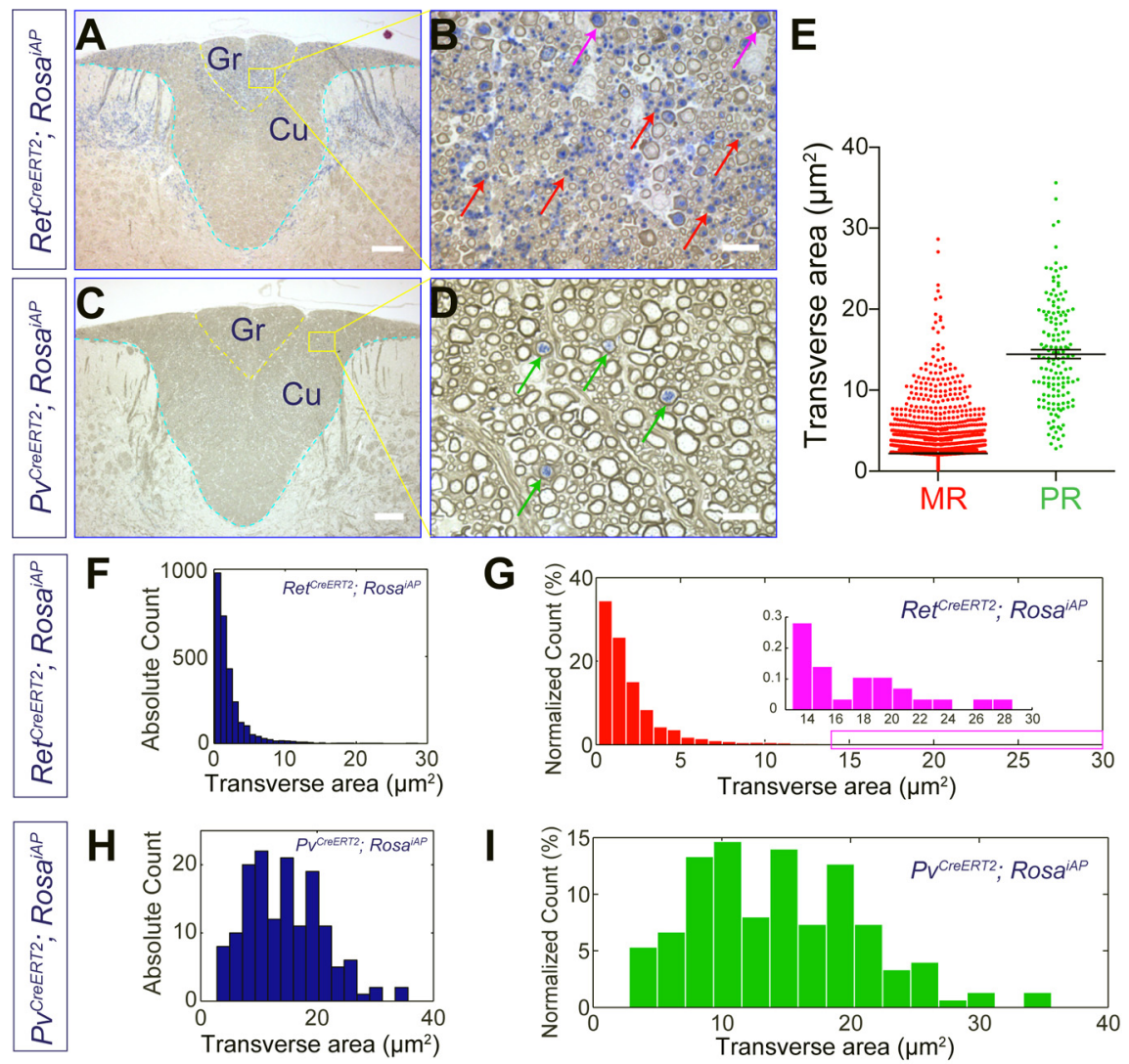

$J$

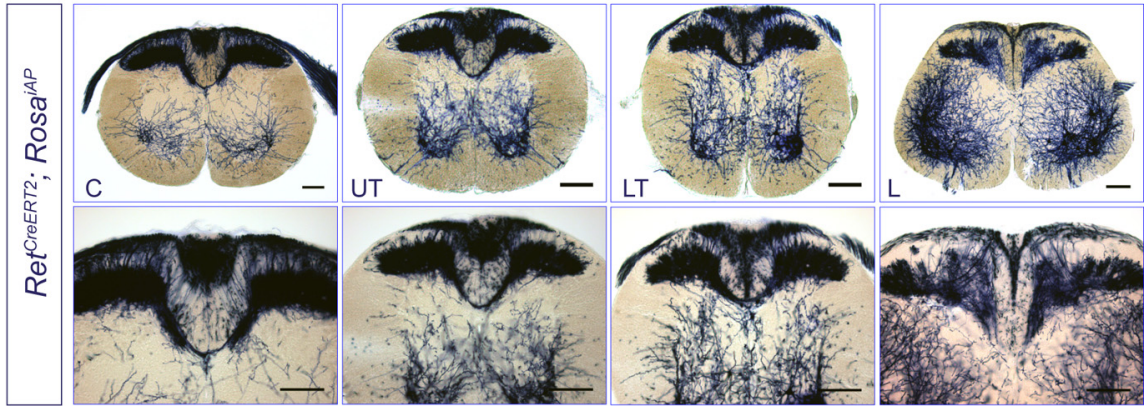

Figure 7. Modality-specific correlation with axonal size in the mouse dorsal column. $A-D$, Semithin sections of adult Ret ${ }^{\text {CreERT2 }}$; Rosa26 $^{i A P}(\boldsymbol{A}, \boldsymbol{B})$ and $P V^{\text {CreERT2 }}$; Rosa26 $6^{i A P}(\boldsymbol{C}, \boldsymbol{D})$ cervical dorsal columns. High magnifications of the boxed areas are shown in $\boldsymbol{B}$ and D. Most RA mechanoreceptors are small-diameter axons (red arrows), whereas few of them are large-diameter axons (magenta arrows). These $\mathrm{AP}^{+}$large-diameter axons are not proprioceptive axons as indicated by their spinal cord innervation pattern $(\boldsymbol{J})$. On the other hand, $\mathrm{AP}^{+}$proprioceptive axons (green arrows) are large-diameter axons found in the lateral area of the dorsal column (D). Scale bars: $\boldsymbol{A}, \boldsymbol{C}, 100 \mu \mathrm{m} ; \boldsymbol{B}, \boldsymbol{D}, 10 \mu \mathrm{m}$. $\boldsymbol{E}$, Comparison of the transverse areas of $\mathrm{AP}^{+}$axons of RA mechanoreceptors and proprioceptors. Error bars indicate SEM; 2840 RA mechanosensory axons and 150 proprioceptive axons were measured. $n=3$ mice for each genotype. $\boldsymbol{F}, \mathbf{G}$, Distribution of transverse areas of labeled RA mechanosensory axons in Ret ${ }^{\text {CreERT2 }}$; Rosa26 $6^{\text {iAP }}$ mice. G, Inset, Distribution of large-diameter RA mechanosensory axons, which make up $<1 \%$ of the totally labeled ones. $\boldsymbol{H}, \boldsymbol{I}$, Distribution of transverse areas of labeled proprioceptive axons in $P V^{\text {CreERT2 }} ; \operatorname{Ros} a 26^{i A P}$ mice. Both absolute count $(\boldsymbol{F}, \boldsymbol{H})$ and percentage are shown $(G, I)$. J, Low- and high-magnification of whole-mount AP color reaction of 6- to 8-week-old Ret ${ }^{\text {CreERT2, }}$;osa26 $6^{\text {iAP }}$ spinal cord sections. $\mathrm{AP}^{+}$fibers highly innervate layers III-V of the spinal cord at different levels, indicating that AP specifically label RA mechanoreceptors. AP also labels motor neurons at the ventral spinal cord, which are also Ret ${ }^{+}$at E11.5 and E12.5. Scale bar, $100 \mu \mathrm{m}$.

this question, we examined semithin sections of cervical spinal cord of rat, feline, canine, rhesus macaque, and human, and lumbar spinal cord of rat, canine, and rhesus macaque (Figs. 10 and 11). We generated heatmaps to characterize the distribution of small- and large-diameter axons in the dorsal column, using the same approach that we developed for mice (Fig. $8 G, H$ ). In all mammalian species we examined, the distribution of small- and large-diameter axons forms a complementary pattern: small-diameter axons are mainly in the medial dorsal column, whereas large-diameter axons are enriched more laterally. Collectively, our results suggest that modality-based organization of ascending somatosensory axons in the DDC pathway is likely to be conserved across multiple mammalian species, including human.

\section{Sparse labeling of RA mechanoreceptors and proprioceptors}

If the mammalian DDC pathway is primarily organized by modality, why has a "somatotopic map" been robustly observed (Whitsel et al., 1970; Smith and Deacon, 1984)? Furthermore, if modalitybased sorting occurs in both gracile and cuneate fasciculi (Fig. $1 C-F$ ), why is only one but not two segregation patterns seen in the rostral spinal cord (Fig. $1 K$ ), where both fasciculi are present? To answer these questions, we examined the central projections of RA mechanoreceptors and proprioceptors at a single-cell level. By titrating different dosage of the CreERT2 ligand $4 \mathrm{HT}$ or tamoxifen, we achieved sparse labeling $(0-1$ neuron labeled per DRG) of RA mechanoreceptors or proprioceptors using $\mathrm{Ret}^{\mathrm{CreERT2}}$; Rosa2 $6^{\mathrm{iAP}}$ and $P v^{\text {CreERT2 }}$; Rosa2 $6^{i A P}$ mice, respectively (see Fig. 13A, B).

We observed a low level of Cremediated background recombination occurring in Ret ${ }^{\text {CreERT2 }}$; Rosa $26^{\text {iAP }}$ mice in the absence of $4 \mathrm{HT}$ treatment, whereas no background recombination was found in the $\mathrm{Pv}^{\mathrm{CreERT2}}$; Rosa2 $6^{\mathrm{iAP}}$ mice. Intriguingly, the $\mathrm{AP}^{+}$DRG neurons from background recombination in $\operatorname{Ret}^{\mathrm{CreERT2}}$; Rosa $26^{i A P}$ mice display different morphologies of central projections: some have long ascending axons projecting through the dorsal column, whereas others project locally (Fig. 12A-C). On average, $25 \pm 3$ DRG neurons were labeled per Ret $^{\text {CreERT2 }}$; Rosa $26^{\text {iAP }}$ mouse at P21, which is composed of $8 \pm 1$ long projecting neurons and $17 \pm 3$ local projecting ones. To distinguish whether this morphological heterogeneity of $\mathrm{AP}^{+}$DRG neurons reflects different subtypes of RA mechanoreceptors (Luo et al., 2009) or different types of Ret ${ }^{+}$DRG neurons (Molliver et al., 1997; Luo et al., 2007), we conducted Fast red-AP color reaction on the spinal cord sections costained with antibodies against CGRP (a marker for peptidergic nociceptors and spinal cord layer I) and VGluT1 (stains the primary afferents of mechanoreceptors, which innervate spinal cord layers III-V). We found that $\mathrm{AP}^{+}$fibers innervate both superficial and deep layers (Fig. 12D-I), suggesting that the background recombination of Ret ${ }^{\text {CreERT2 }}$; Rosa $26^{\text {AAP }}$ mice occurs in both RA 

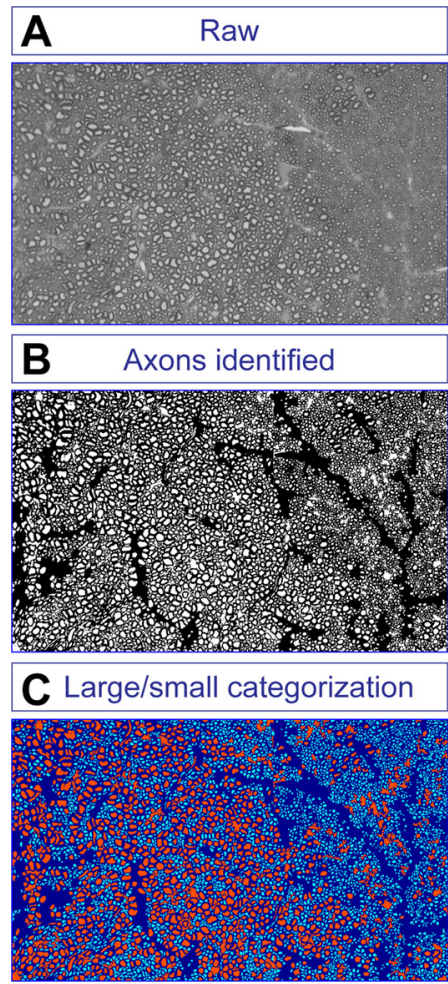

D Density of small axons

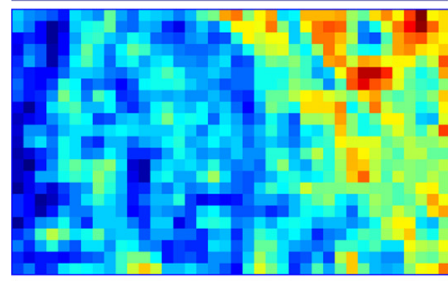

E Density of large axons

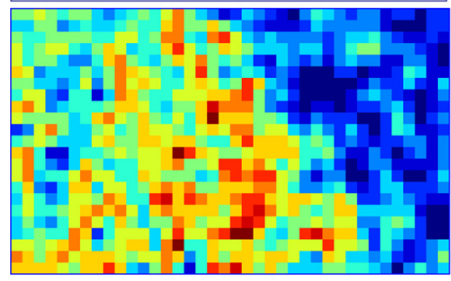

$\mathbf{F}$

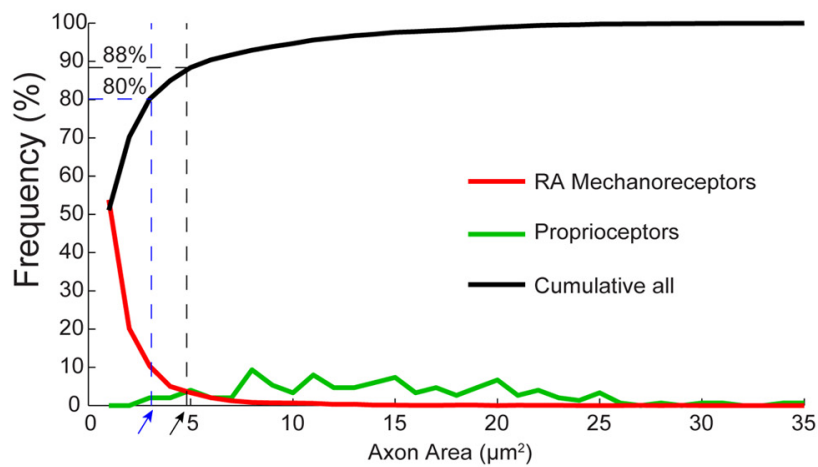

$\mathbf{G}$

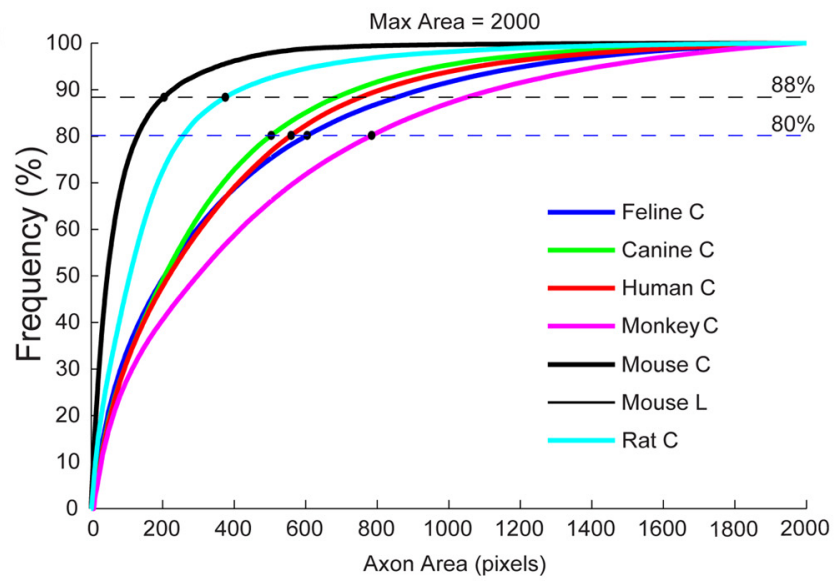

H

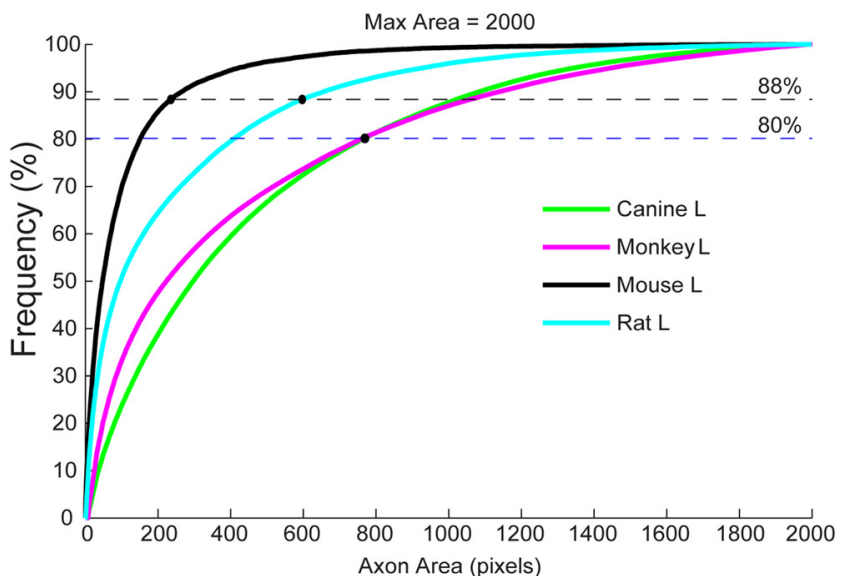

Figure 8. Method of generating heatmaps to represent the density of small- and large-diameter dorsal column fibers. $\boldsymbol{A}-\boldsymbol{E}, A$ computer program was written and run in MATLAB to automatically calculate the axonal size and density in the dorsal column. $\boldsymbol{A}$, An example raw image cropped from a mouse sample (current size, $500 \times 300$ pixels). $\boldsymbol{B}$, Binary image showing areas identified as axons (white). C, Image showing categorization of axons as large (red) and small (cyan) with the $88 \%$ as the category threshold. $\boldsymbol{D}$, Heatmap of large axon density in sliding windows. $\boldsymbol{E}$, Heatmap of small axon density in sliding windows. $\boldsymbol{F}$, Probability density functions for AP labeled mechanosensory axons (red trace) and proprioceptive axons (green) and cumulative distribution function for all dorsal column axons (black). Arrows indicate axon area values corresponding to 1 st and 5 th percentile of proprioceptor axons, which correspond to 80th and 88th percentile of all axons, respectively. $\boldsymbol{G}, \boldsymbol{H}$, Cumulative distribution functions for all MATLAB-extracted axons of cervical $(\boldsymbol{G})$ and lumbar $(\boldsymbol{H})$ spinal cord sections of different mammalian species.

mechanoreceptors and other types of $\operatorname{Ret}^{+}$DRG neurons (late Ret $^{+}$DRG neurons), likely including nociceptors and C-LTMRs. Based on the known morphologies of RA mechanoreceptors, nociceptors, and C-LTMRs (Brown, 1981; Ling et al., 2003; Li et al., 2011), we hypothesized that $\mathrm{AP}^{+}$DRG neurons with long ascending axons are RA mechanoreceptors, and those with local central projections are late Ret $^{+}$DRG neurons. If this hypothesis is true, we predict that the ratio of $\mathrm{AP}^{+}$long projecting neurons versus local projecting ones in nontreated Ret $^{\text {CreERT2 }}$; Rosa $26^{i A P}$ mice would decrease from P0 to P21 because more DRG neurons turn on the expression of Ret postnatally and thus CreERT2 ac- tivity increases in late Ret $^{+}$DRG neurons (Molliver et al., 1997; Luo et al., 2007). In addition, this ratio should be reversed upon 4 HT treatment before E13.5, which specifically activates CreERT2 in RA mechanoreceptors (Luo et al., 2009). Indeed, we found that the percentage of long projecting neurons significantly decreases from P0 to P21 (80.1 $\pm 4.4 \%, \mathrm{P} 0 ; 34.4 \pm 3.3 \%$, $\mathrm{P} 21 ;{ }^{\star} p<0.001$, Fig. $\left.12 \mathrm{~J}\right)$, whereas the percentage of local projecting ones increases accordingly (19.9 $\pm 4.4 \%$, P0; $65.6 \pm 3.3 \%$, $\mathrm{P} 21 ;{ }^{*} p<0.001$, Fig. $\left.12 \mathrm{~J}\right)$. This trend was reversed upon the $4 \mathrm{HT}$ treatment at E12.5 (long, $73.9 \pm 2.4 \%$; local, $26.1 \pm 2.4 \%$, P21; ${ }^{\star} p<0.001$, Fig. 12J). Furthermore, we costained $\mathrm{AP}^{+}$DRG neu- 
A
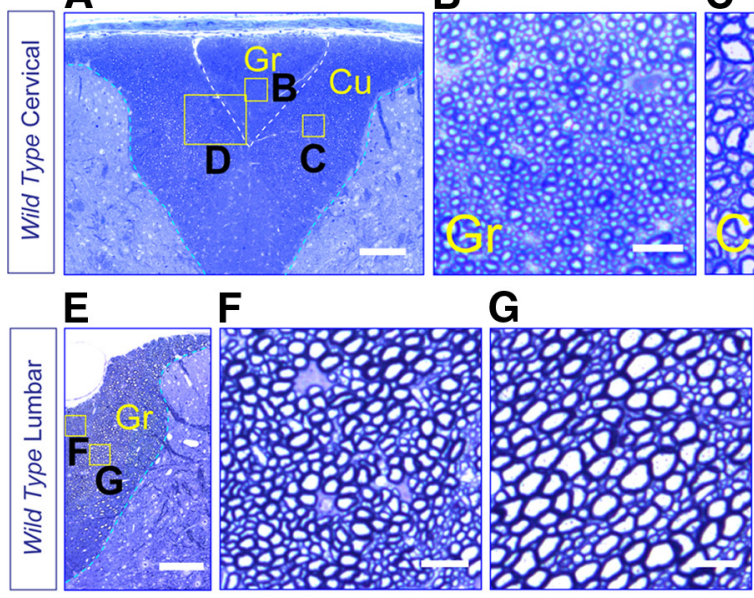

C
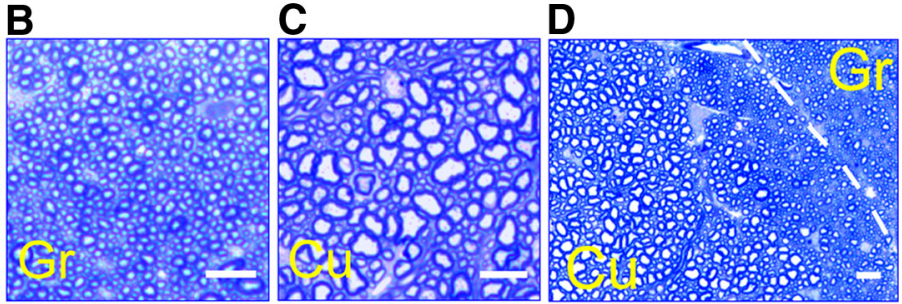
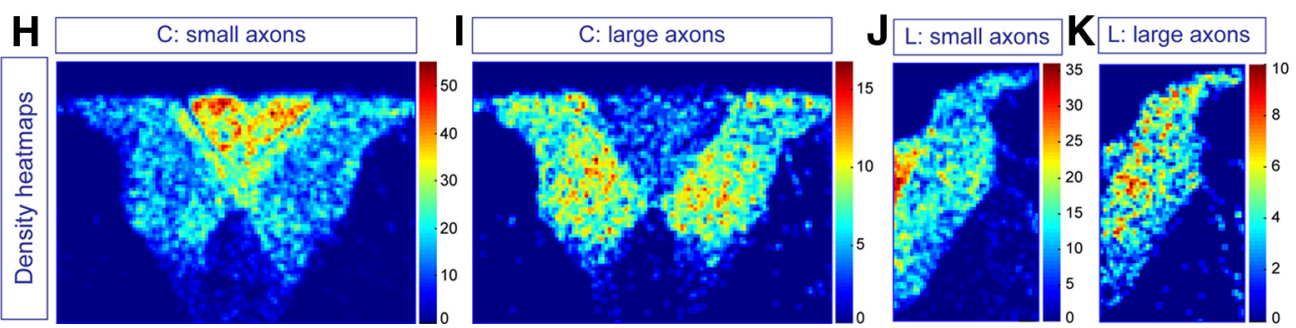

Figure 9. Modality segregation revealed by distribution of small- and large-diameter axons in the mouse dorsal column. $\boldsymbol{A}-\boldsymbol{G}$, Semithin sections of the cervical $(\boldsymbol{A}-\boldsymbol{D})$ and lumbar dorsal column $(\boldsymbol{E}-\boldsymbol{G})$ of a 3 month WT mouse. Dashed white lines indicate the boundary between $\mathrm{Gr}$ and $\mathrm{Cu}$; dashed cyan linesindicate the edge between the dorsal column and the dorsal horn. $A-C$, Overview of the cervical dorsal column $(\boldsymbol{A})$, and higher magnified images of the boxed area in gracile $(\boldsymbol{B})$, cuneate fasciculus $(\boldsymbol{C})$, and the boundary areas of $\mathrm{Gr}$ and $\mathrm{Cu}(\boldsymbol{D})$. $\boldsymbol{E}-\boldsymbol{G}, 0$ verview of the lumbar dorsal column $(\boldsymbol{E})$ and higher-magnification images in the medial $(\boldsymbol{F})$ and lateral $(\boldsymbol{G})$ part of the Gr. Scale bars: $\boldsymbol{A}, \boldsymbol{E}, 100 \mu \mathrm{m} ; \boldsymbol{B}-\boldsymbol{D}, \boldsymbol{F}, \boldsymbol{G}, 10 \mu \mathrm{m}$. $\boldsymbol{H}-\boldsymbol{K}$, Heatmaps showing the density of small-and large-diameter axons. $\boldsymbol{H}, \boldsymbol{I}$, In the cervical dorsal column, small-diameter axons are enriched in the medial gracilefasciculus and the medial marginal zone of the cuneatefasciculus, whereas large-diameter axons are mainly in the lateral cuneate fasciculus.J, $\boldsymbol{K}$, At lumbar level, small-diameter axons are mainly in the medial gracile fasciculus whereas large-diameter axons are more laterally located.

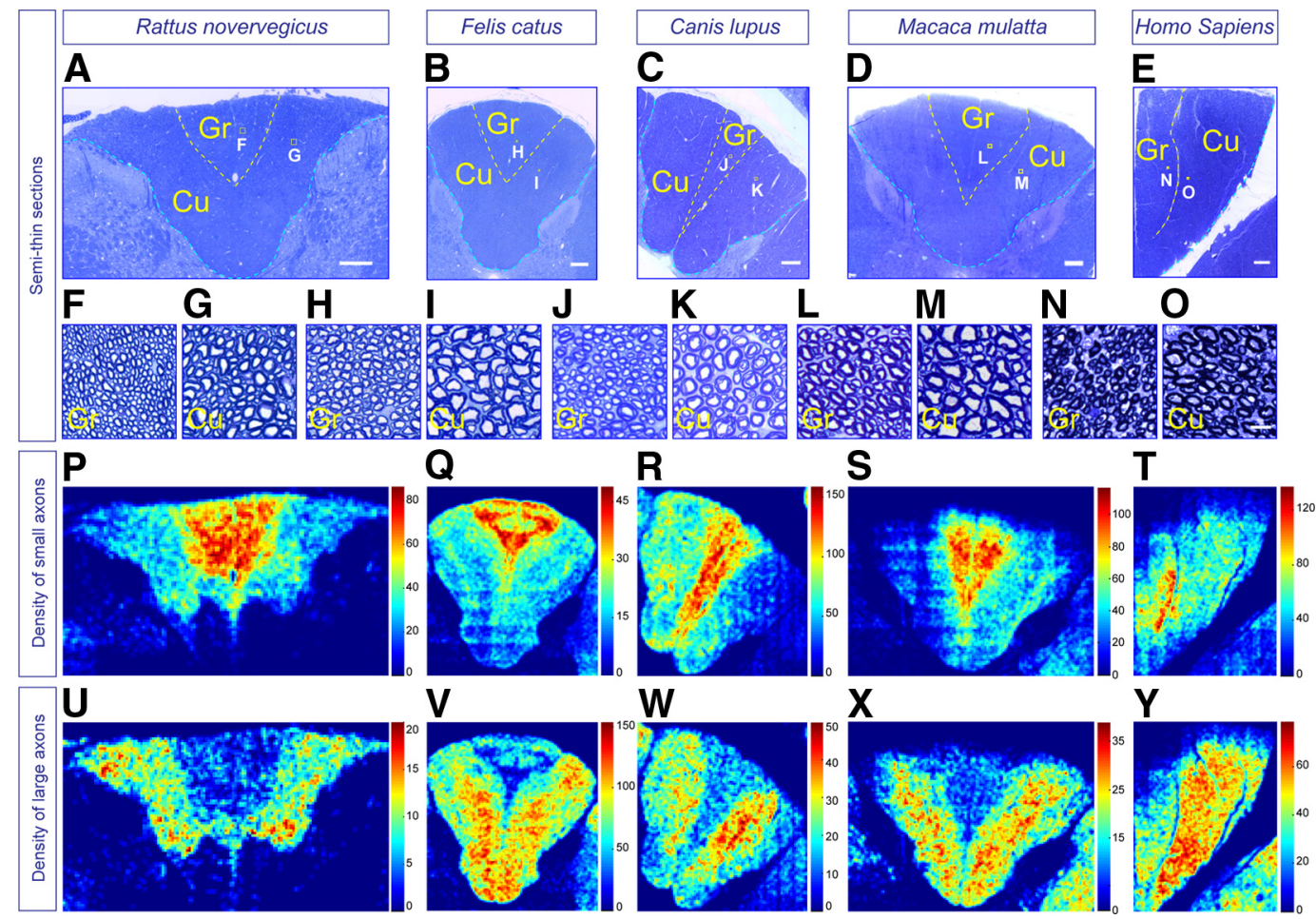

Figure 10. Modality-based organization in the cervical dorsal column of multiple mammalian species. $\boldsymbol{A}-\boldsymbol{E}$, The semithin sections of rat $(\boldsymbol{A})$, feline $(\boldsymbol{B})$, canine $(\boldsymbol{C})$, monkey $(\boldsymbol{D})$, and human $(\boldsymbol{E})$ cervical dorsal column. $\boldsymbol{F}-\mathbf{O}$, Higher-magnification images of boxed areas in $\boldsymbol{A}-\boldsymbol{E} . \boldsymbol{M}-\boldsymbol{P}$, Heatmaps showing the density of small $(\boldsymbol{P}-\boldsymbol{T})$ and large $(\boldsymbol{U}-\boldsymbol{Y})$ axons of the cervical dorsal column of each species. Dashed yellow lines indicate the boundary between gracile and cuneate fasciculi; dashed cyan lines indicate the edge between the dorsal column and the dorsal horn. Scale bars: $\mathbf{A}, \boldsymbol{B}, 200 \mu \mathrm{m} ; \mathbf{C}-\boldsymbol{E}, 300 \mu \mathrm{m} ; \boldsymbol{F}-\mathbf{O}, 10 \mu \mathrm{m}$. 


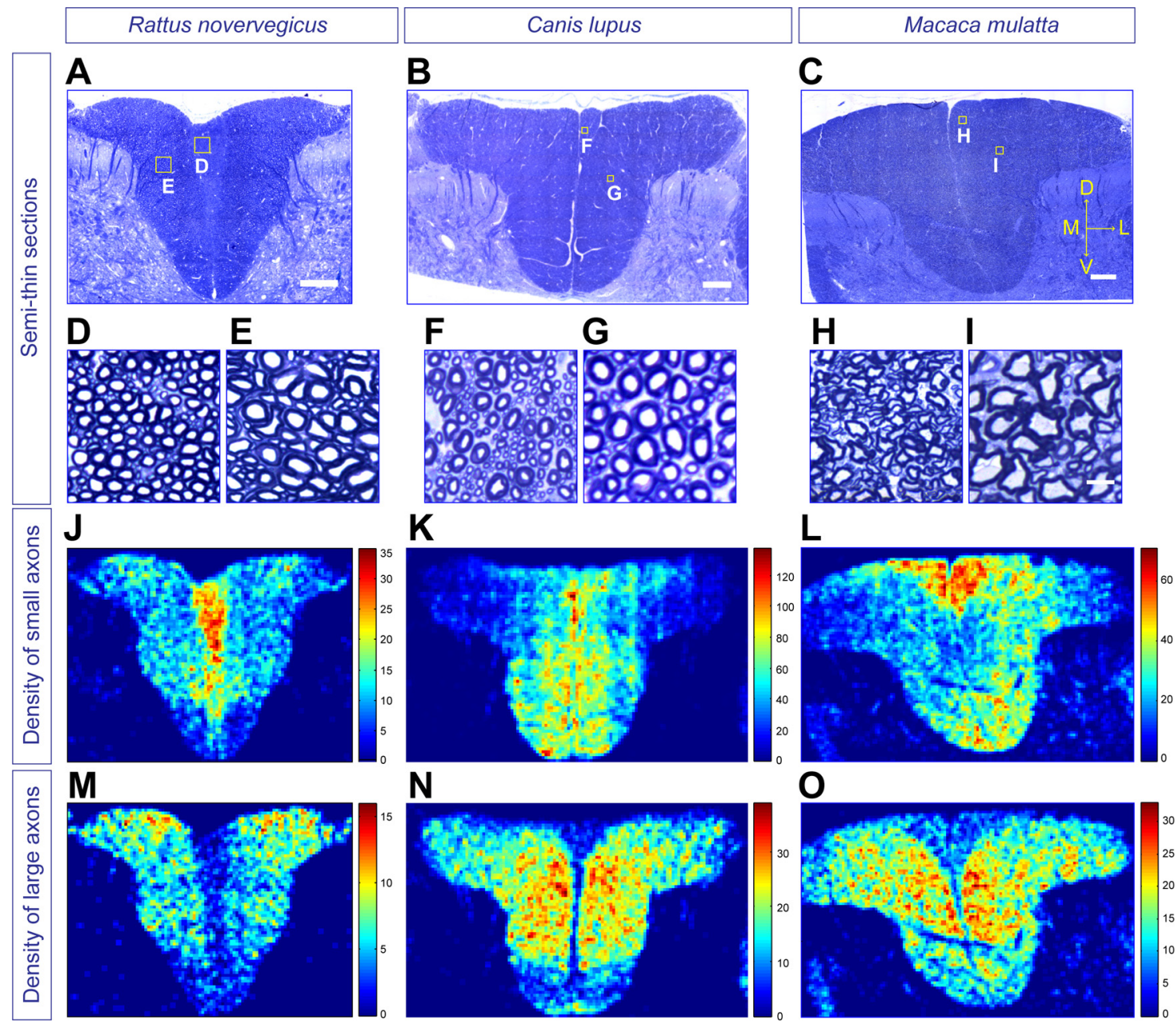

Figure 11. Axon distribution reveals modality-based organization in the lumbar dorsal column of multiple mammalian species. $\boldsymbol{A}-\boldsymbol{C}$, The semithin sections of rat $(\boldsymbol{A})$, canine $(\boldsymbol{B})$, and monkey $(\boldsymbol{C})$ lumbar dorsal column (stained with toluidine blue). $\boldsymbol{D}-\boldsymbol{I}$, Higher-magnification images of boxed areas in $\boldsymbol{A}-\boldsymbol{C}$, showing the axonal size difference between medial and lateral gracile fasciculus of each species. $\boldsymbol{J}-\mathbf{0}$, Heatmaps show the density of small $(\boldsymbol{J}-\boldsymbol{L})$ and large $(\boldsymbol{M}-\mathbf{0})$ axons of the lumbar dorsal column of each species. Scale bars: $\boldsymbol{A}, 100 \mu \mathrm{m} ; \boldsymbol{B}, 200 \mu \mathrm{m}$; C, $300 \mu \mathrm{m} ; \mathbf{D}-I, 10 \mu \mathrm{m}$.

rons with NFH antibody (Fig. $12 \mathrm{~K}-\mathrm{P}$ ), which stain the myelinated RA mechanoreceptors but not late $\operatorname{Ret}^{+}$DRG neurons. Consistently, we found that the percentage of $\mathrm{AP}^{+} / \mathrm{NFH}^{+}$ neurons decreases $(70.3 \pm 3.6 \%, \mathrm{P} 0 ; 43.2 \pm 0.05 \%, \mathrm{P} 21 ; p<$ $0.05)$, whereas the percentage of $\mathrm{AP}^{+} / \mathrm{NFH}^{-}$neurons increases from P0 to P21 (29.7 $\pm 3.6 \%$, P $; 56.8 \pm 0.05 \%$, P 21 ; $p<0.05$, Fig. 12Q), and this change is also reversed upon early $4 \mathrm{HT}$ treatment $\left(\mathrm{AP}^{+} / \mathrm{NFH}^{+}, 64.8 \pm 0.5 \% ; \mathrm{AP}^{+} / \mathrm{NFH}^{-}\right.$, $26.1 \pm 0.5 \%, \mathrm{P} 21 ; p<0.001$, Fig. 12Q). Together, we conclude that $\mathrm{AP}^{+}$long projecting neurons in nontreated Ret $^{\text {CreERT2 }}$; Rosa26 ${ }^{\text {iAP }}$ mice are RA mechanoreceptors and took advantage of the background recombination to visualize central projections of individual mechanoreceptors.

Key morphological differences between ascending axons of mechanoreceptors and proprioceptors

We dissected out the whole spinal cord with attached DRGs from 3-week-old Ret ${ }^{\mathrm{CreERT}}$; Rosa26 $6^{\mathrm{iAP}}$ or $\mathrm{Pv}^{\mathrm{CreERT2}}$; Rosa26 $6^{\mathrm{iAP}}$ mice and conducted AP color reaction. Using this whole-mount preparation, we could trace the entire central projections of labeled mechanoreceptors and proprioceptors. Both RA mechanoreceptors and proprioceptors have a typical pseudo-unipolar morphology: a single axon bifurcates near the soma, with one branch projecting centrally toward the spinal cord and the other to the periphery (Fig. 13A,B). The central axons of mechanoreceptors and proprioceptors bifurcate again upon entering the spinal cord, generating the rostrally (ascending) and caudally (descending) projecting branches that grow interstitial third-order collaterals. Interestingly, we observed several key morphological differences between the afferents of mechanoreceptors and proprioceptors in the dorsal column. First, ascending RA mechanosensory axons usually travel 3-4 spinal cord segments rostrally in the dorsal lateral spinal cord and give off collaterals before entering the dorsal column, whereas ascending proprioceptive axons join the dorsal column within one segment and give off collaterals along their entire length (Fig. $13 C, D)$. Second, after joining the dorsal column, ascending RA mechanosensory axons gradually move to the middle of the dorsal column and project rostrally, whereas proprioceptive axons ascend in the lateral dorsal column (Figs. 13 E, F and Fig. 14). Thus, ascending RA mechanosensory axons cross proprioceptive axons to enter the middle of the dorsal column, which explains the little bit "mix" of these two modalities at the cross section (Figs. 1 and 6). Third, ascending RA mechanosensory axons terminate in the DCN, whereas proprioceptive axons from caudal DRGs terminate in the dorsal 

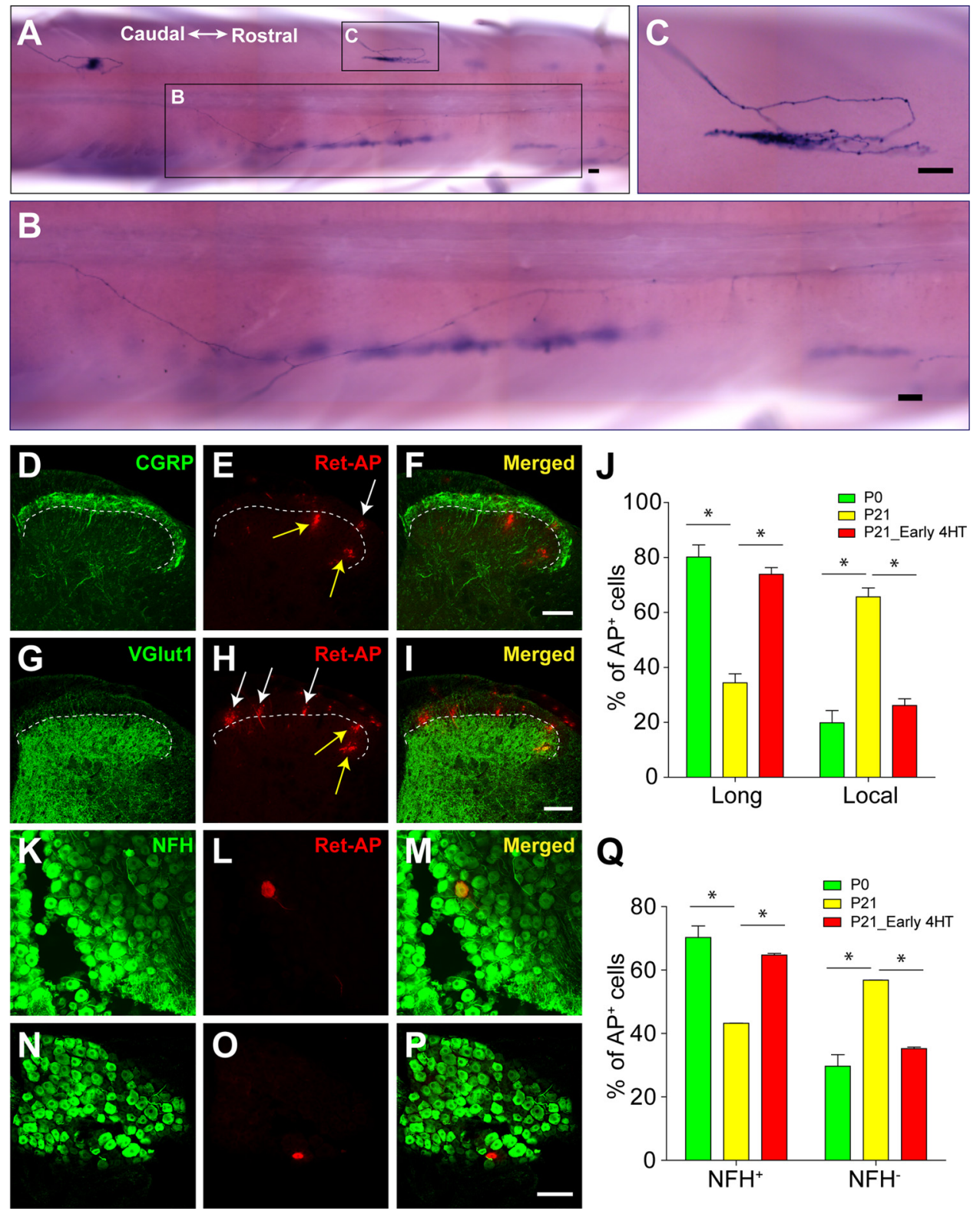

Figure 12. Background recombination of Ret ${ }_{\text {CreRT2}}$; Rosa2 $6^{\text {iAP }}$ mice. $A-C$, Whole-mount AP color reaction of the P21 Ret ${ }^{\text {CreERT2 }}$; Rosa2 $6^{\text {iAP }}$ spinal cord without $4 \mathrm{HT}$ treatment showing that two types of morphologically different neurons are labeled by AP. One type of neurons grows long projections $(\boldsymbol{B})$, which bifurcate at the dorsal root entry zone and send long ascending and descending axons in the dorsal column, and the other ones are locally projecting neurons (C).D-I, Immunostaining of CGRP or VGluT1 of adult Ret ${ }^{\text {TreERT2 }}$; Rosa2 $6^{\text {iAP }}$ spinal cord sections combined with Fast-red $\mathrm{AP}$ color reaction. $\mathrm{AP}^{+}$fibers innervate different dorsal horn layers. $\mathbf{D}-\boldsymbol{F}$, Dashed white lines indicate the boundary between layers I and II defined by CGRP staining. G-I, Dashed white lines indicate the boundary between layers II and III, defined by VGluT1 staining. $\boldsymbol{E}, \boldsymbol{H}$, White arrows point to the $\mathrm{AP}^{+}$fibers that innervate superficial layers; yellow arrows indicate the AP ${ }^{+}$fibers that innervate

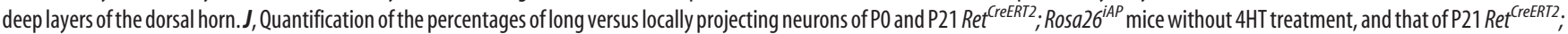
Rosa26 ${ }^{\text {iAP }}$ mice with $0.25 \mathrm{mg} 4 \mathrm{HT}$ treatment atE12.5. K-P, Whole-mount DRG immunostaining of anti-NFH and Fast-red AP color reaction. $M$, An example of NFH ${ }^{+} / \mathrm{AP}^{+} \mathrm{DRG}$ neuron. $P$, An example of $\mathrm{NFH}^{-} / \mathrm{AP}^{+} \mathrm{DRG}$ neuron. Scale bar, $100 \mu \mathrm{m} . \mathbf{Q}$, Quantification of the percentages of $\mathrm{NFH}^{+} / \mathrm{AP}^{+}$and NFH ${ }^{-} / \mathrm{AP}^{+}$DRG neurons of P0 and P21 Ret ${ }^{\text {CreeRT2 }}$; Rosa2 $6^{\text {iAP }}$ mice without $4 \mathrm{HT}$ treatment, and that of P21 Ret ${ }^{\text {CreeRT2 }}$; Rosa2 $6^{\text {iAP }}$ mice with $0.25 \mathrm{mg} 4 \mathrm{HT}$ treatment at E12.5. Error bars indicate SEM. $n=6-8$ mice. $^{*} p<0.001$.

column and grow short processes innervating the intermediate spinal cord (Fig. 13F). Fourth, as a result of axon sorting in the dorsal column, ascending RA mechanosensory and proprioceptive axons innervate distinct domains of the DCN (Fig. $13 G, H)$. In total, we examined 100 RA mechanoreceptors and 135 proprioceptors (Fig. 13I,J) and found that almost all ascending RA mechanosensory afferents, regardless of their soma location, innervate the medulla. In contrast, ascending proprioceptive axons from $\mathrm{T} 6$ and above reach the medulla, whereas those below T6 travel 4-15 segments and then terminate.

\section{D reconstruction of sparsely traced $\mathrm{RA}$ mechanoreceptors} and proprioceptors

To thoroughly characterize the morphologies of the entire central projections of RA mechanoreceptors and proprioceptors, we also prepared montages from sparsely labeled Ret ${ }^{\text {CreERT2}} ; \operatorname{Ros} 26^{i A P}$ and $P v^{\text {CreERT2 }}$; Rosa $26^{\text {iAP }}$ spinal cords, using multiple focal planes for 3D reconstruction (Fig. 14). Based on the collateral morphologies, we have both $\mathrm{Ia}$ (Fig. $14 F-J$ ) and Ib (Fig. $14 K-N, S-W$ ) proprioceptors traced in $P v^{\text {CreERT2 }}$; Rosa $26^{i A P}$ mice. Similar to what was previously reported (Brown, 1981), Ia proprioceptors give off both short and long collaterals, which innervate the Clarke's column and motor 

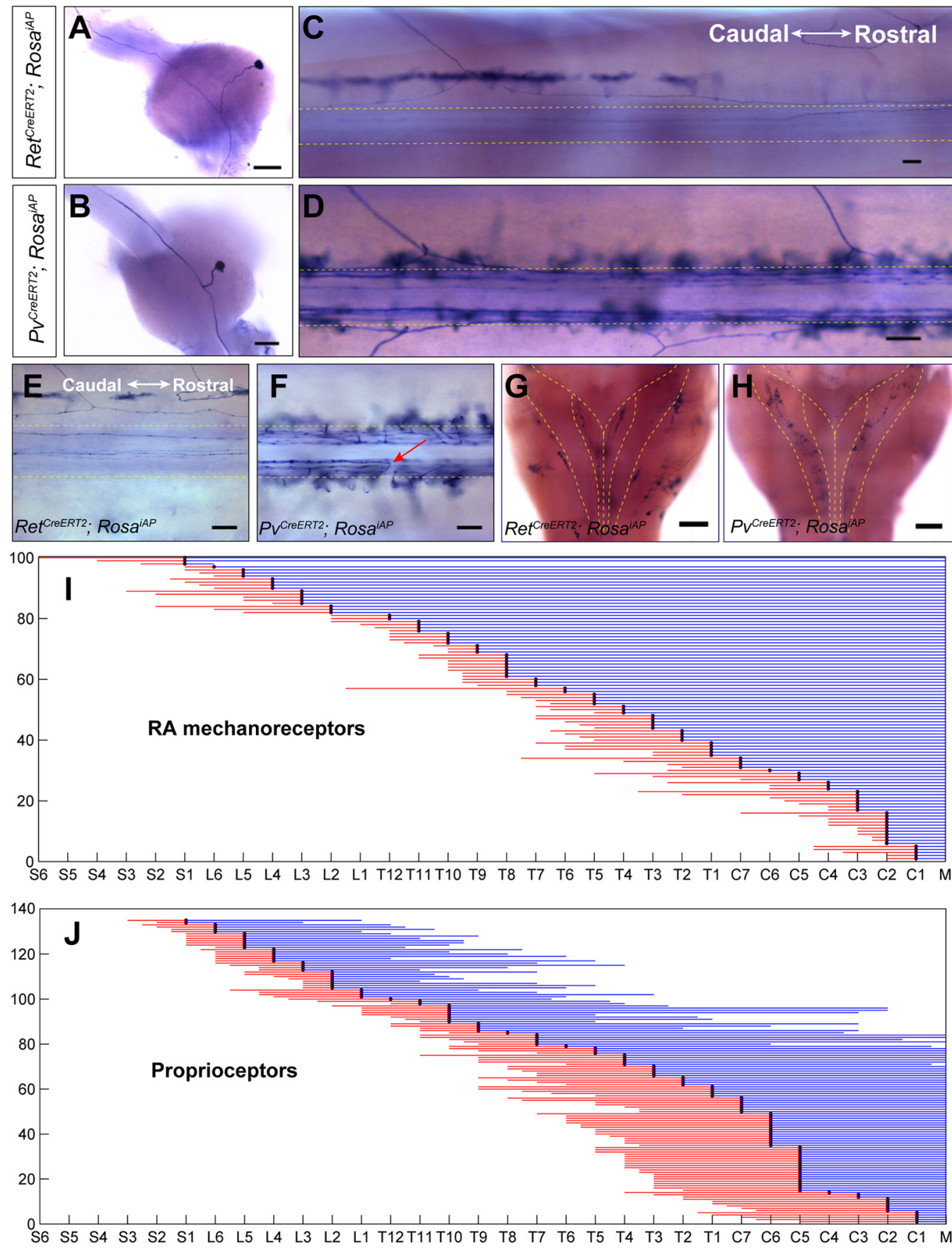

Figure 13. Sparse labeling of RA mechanoreceptors and proprioceptors uncovers key morphological differences. $A, B$, AP color reaction of whole-mount Ret ${ }^{\text {CreERT2 }}$; Rosa2 $6^{\text {iAP }}$ and $P V^{\text {CreERT2 }}$; Rosa2 $6^{\text {iAP }}$ DRGs, showing one labeled RA mechanoreceptor $(\boldsymbol{A})$ and proprioceptor $(\boldsymbol{B})$, respectively. $\boldsymbol{C}, \boldsymbol{D}$, Dorsal view of the whole-mount spinal cord shows the central projection of RA mechanoreceptors and proprioceptors. After bifurcation, one lumbar RA mechanoreceptor travels 3-4 segments before joining the dorsal column pathway $(\boldsymbol{C})$, whereas proprioceptors enter the dorsal column within the same segment $(\boldsymbol{D}) . \boldsymbol{E}, \boldsymbol{F}, \mathrm{AP}^{+}$ascending axons of RA mechanoreceptors $(\boldsymbol{E})$ and proprioceptors $(\boldsymbol{F})$ travel in the dorsal column. $\boldsymbol{F}$, Red arrow indicates the endpoint of a proprioceptive axon. $\mathbf{C}-\boldsymbol{F}$, Images are oriented as caudal (left) and rostral (right). $\boldsymbol{G}, \boldsymbol{H}$, Dorsal views of the medulla show the different termination domains of RA mechanoreceptors $(\boldsymbol{G})$ and proprioceptors $(\boldsymbol{H})$ in the DCNs. Dashed yellow lines outline the DCN areas. Scale bar, $100 \mu \mathrm{m} . \boldsymbol{I}, \boldsymbol{J}$, Summary of ascending and descending axons of 100 RA mechanoreceptors from 14 mice $(I)$ and 135 proprioceptors from 13 mice $(J)$. Black dots indicate where the cell bodies are located; blue and red lines indicate the ascending and descending fibers respectively. Almost all RA mechanoreceptors reach medulla regardless of their cell body locations, whereas only proprioceptors from DRGs above T6 reach medulla.

neurons, respectively (Fig. 14H). The 3D reconstructions of RA mechanoreceptors and proprioceptors confirm the morphological differences between their ascending axons.

\section{Lesion study confirms that ascending axons of lumbar} proprioceptors do not reach rostral dorsal column To further confirm that ascending proprioceptive axons from caudal DRGs do not reach rostral dorsal column, we performed lumbar dorsal root rhizotomy, which causes the degeneration of ascending axons from the injured segments and examined the degeneration pattern at L2, T6, and C3 levels. One to 2 weeks after dorsal rhizotomy, degenerating axons can be identified in the dorsal column semithin sections by their dark staining and irregular morphologies. These degenerating fibers are found in the gracile fasciculus at all levels examined (Fig. 15A-C). Interestingly, the number of degenerating axons resulting from the lumbar lesion greatly decrease at $\mathrm{T} 6$ 


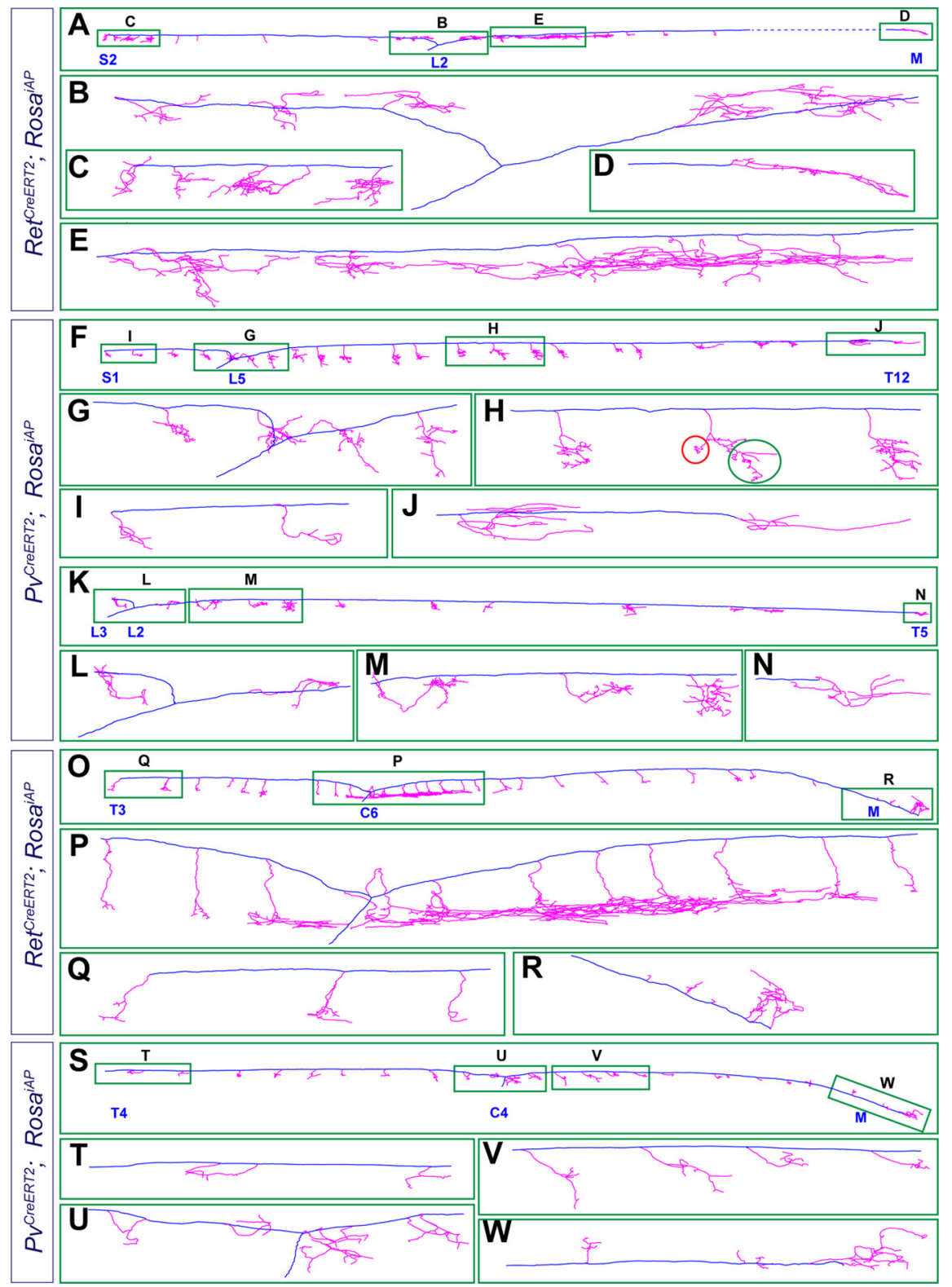

Figure 14. 3D reconstruction of genetically traced RA mechanoreceptors and proprioceptors. Blue lines indicate the first- and secondorder central projections; magenta lines indicate the interstitial collaterals innervating the spinal cord or DCNs. $\boldsymbol{A}-\boldsymbol{E}, \mathrm{A}$ representative example of $3 \mathrm{D}$ reconstructed lumbar RA mechanoreceptors and of P21 Ret ${ }^{\mathrm{CreERT}}$; Rosa $26^{\mathrm{iAP}}$ mice. $A$, Overview of the L2 RA mechanoreceptor, with an ascending axon ending at medulla and a descending axon stopping at $\$ 2$. The ascending axon sends several collaterals to the dorsal spinal cord before it enters the dorsal column at the T10 level but grows no collaterals afterward (represented by dashed blue line) until it reaches medulla. The descending axon also grows a few collaterals. Enlarged view of the bifurcation area and collaterals near the dorsal root entry zone $(\boldsymbol{B})$, collaterals near the descending endpoint $(\boldsymbol{C})$, the nerve endings in the medulla $(\boldsymbol{D})$, and the collaterals arising from the ascending axon $(\boldsymbol{E})$ are also shown. $\boldsymbol{F}-\boldsymbol{N}$, Representative examples of $3 \mathrm{D}$ reconstructed lumbar group la $(\boldsymbol{F}-\boldsymbol{J})$ and $\mathrm{Ib}(\boldsymbol{K}-\boldsymbol{N})$ proprioceptors of $\mathrm{P} 21 \mathrm{Pv}^{\mathrm{CreERT}}$; Rosa2 ${ }^{\text {iAP }}$ mice. $\boldsymbol{F}$, Overview of an $\mathrm{L} 5$ group la proprioceptor, with an ascending fiber ending at $\mathrm{T} 12$ and a descending fiber stopping at $\$ 1$. Enlarged view of different areas are also shown, including the bifurcation area $(\boldsymbol{G})$, collaterals innervating the intermediate area (red circle) and those innervating ventral horn (green ellipse) of the spinal cord $(\boldsymbol{H})$, descending endpoint at $S 1(\boldsymbol{I})$, and the ascending endpoint at T12 $(\boldsymbol{J})$. $\boldsymbol{K}$, Overview of an L2 group Ib proprioceptor, which has an ascending fiber ending at T5 and a descending fiber stopping at $L 3$. Enlarged view of the bifurcation area and descending endpoint $(\boldsymbol{L})$, the collaterals arising from the main afferent to show the innervation of the intermediate area in the spinal cord $(\boldsymbol{M})$, and the ascending endpoint at $\mathrm{T5}(\boldsymbol{N})$ are also shown. Both group la and $\mathrm{lb}$ fibers from the lumbar levels drop out of the ascending pathway with a terminal innervating interstitially in the spinal cord. $\mathbf{0}-\boldsymbol{W}$, Representatives of 3D reconstructed cervical RA mechanoreceptor $(\mathbf{O}-\boldsymbol{R})$ and proprioceptor $(\boldsymbol{S}-\boldsymbol{W})$. Both havetheir ascending fibers ending at medulla.

and C3 levels (Fig. 15D), indicating that some ascending axons from L4-L5 do not reach the rostral dorsal column. Based on morphologies of axons in the matching area of the contralateral side, we deduce that axons from L4-L5 proprioceptors drop out of the gracile fascic- ulus because most of the remaining degenerating axons at T6 and C3 are smalldiameter axons of mechanoreceptors (Fig. $15 A-C)$.

\section{Somatotopic organization within the same modality}

To examine the somatotopic relationship among ascending axons of the same modality, we replotted the relative position of $\mathrm{AP}^{+} \mathrm{RA}$ mechanosensory afferents in the Ret ${ }^{\text {CreERT2}^{2}}$; Rosa $26^{\text {iAP }}$ dorsal column at C3 level (Fig. 16A), based on their best focal planes within the $\mathrm{Z}$ serials of images. We found that ascending axons of caudal to rostral RA mechanoreceptors display a medial to lateral pattern in the dorsal column. This result suggests that a somatotopic organization exists within the same modality (intramodality somatotopic organization).

\section{The new modality-based functional organization model of DDC pathway} Our results enable us to propose a new model to explain how somatotopic and modality-based organizations coexist in the DDC pathway (Fig. 16B): (1) ascending somatosensory axons from DRGs below and above T6 travel in the gracile and cuneate fasciculi, respectively; and (2) upon entering the dorsal column, RA mechanosensory afferents tend to travel in a more medial position than proprioceptive axons, which leads to the modality-based organization within each fasciculus. In addition, almost all proprioceptive afferents from sacral to lower thoracic DRGs terminate in the rostral dorsal column. This leaves RA mechanosensory afferents in the rostral gracile fasciculus where the cuneate fasciculus forms, resulting in the observed single modality-based organization pattern in the rostral dorsal column. (3) Ascending RA mechanosensory and proprioceptive axons from the same DRG enter the dorsal column at different segments. Thus, at a given segment, medial axons are RA mechanosensory axons from more caudal DRGs, whereas lateral axons are proprioceptive axons from more rostral DRGs. In combination with somatotopic organization within each modality, a rough "somatotopic map" is generated in the dorsal column (Fig. 16C); and (4) as a result of axon sorting in the dorsal column, these modality-based and somatotopic organizations of mechanosensory and proprioceptive axons are preserved in the DCNs.

\section{Discussion}

Although the current dominant view suggests that the DDC pathway is organized as a "somatotopic map," we demonstrated that RA mechanosensory and proprioceptive afferents are largely segregated in the mouse dorsal column. This 
modality-based organization is likely to be conserved in multiple mammalian species, including human. We also identified several key morphological differences between RA mechanosensory and proprioceptive ascending axons, which leads to modality segregation in the DDC pathway and explains the previous findings of a "somatotopic map." Together, our results establish a new functional organization model for the mammalian DDC pathway.

\section{Modality-based functional organization in the DDC pathway}

Spinal cord dorsal column is one of the best known examples of the "somatotopic organization." It is widely accepted that caudal to rostral ascending somatosensory axons are organized in a medial to lateral pattern in the dorsal column (Smith and Deacon, 1984; Charles Watson, 2009). Because several different types of somatosensory afferents join the dorsal column from each spinal cord segment, this "somatotopic map" model predicts that they would intermingle in the dorsal column. Whitsel et al. carefully compared afferents in the lumbar and cervical gracile fasciculus of squirrel monkey with regard to both modality and receptive field (Whitsel et al., 1969, 1970). They found that afferents of proprioceptors and A $\beta$-LTMRs were organized as a dermatomal map at lumbar levels, whereas only RA mechanoreceptors were retained in the cervical gracile fasciculus. They proposed that gracile fasciculus somehow "re-sort" while projecting rostrally, presumably because ascending axons of proprioceptors and slowly adapting (SA) LTMRs from caudal DRGs do not reach the cervical dorsal column.

On the other hand, physiological recordings also suggest that somatosensory fibers carrying the same modality of information project together in the dorsal column (Uddenberg, 1968) and innervate distinct domains of DCNs (Dykes et al., 1982; Hummelsheim et al., 1985; Fyffe et al., 1986). However, this modalitybased functional organization of the dorsal column has not become a dominant view because no direct supportive anatomical evidence is currently available. Although the spinal cord and DCN innervating collaterals of $\mathrm{A} \beta$ mechanoreceptors and proprioceptors were well studied (Brown, 1981; Fyffe et al., 1986), the structures of their ascending axons through the entire dorsal column have not been carefully examined.

Using modality-specific genetic tracing, here we provide the first systematic characterization of mechanosensory and proprioceptive ascending axons in the mammalian dorsal column. With a whole-mount spinal cord preparation and AP color reaction, which enable us to visualize the entire ascending axons of individual mechanoreceptors and proprioceptors, we demonstrate that ascending axons of mouse RA mechanroeceptors and proprioceptors, two major types of ascending fibers in the DDC pathway, are largely segregated from each other throughout the entire dorsal column (Figs. 1, 2, and 6). This finding cannot be explained by the "somatotopic model" or even the "re-sorting"

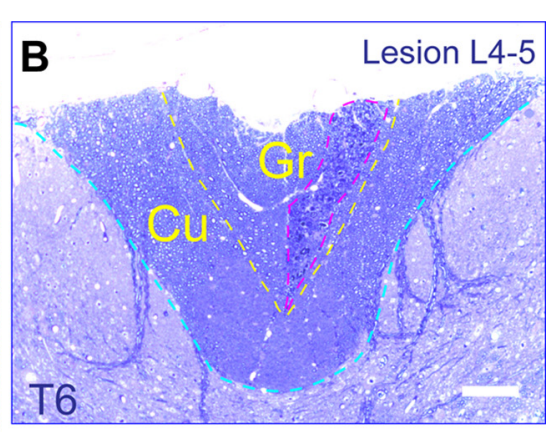

Lesion L4-5
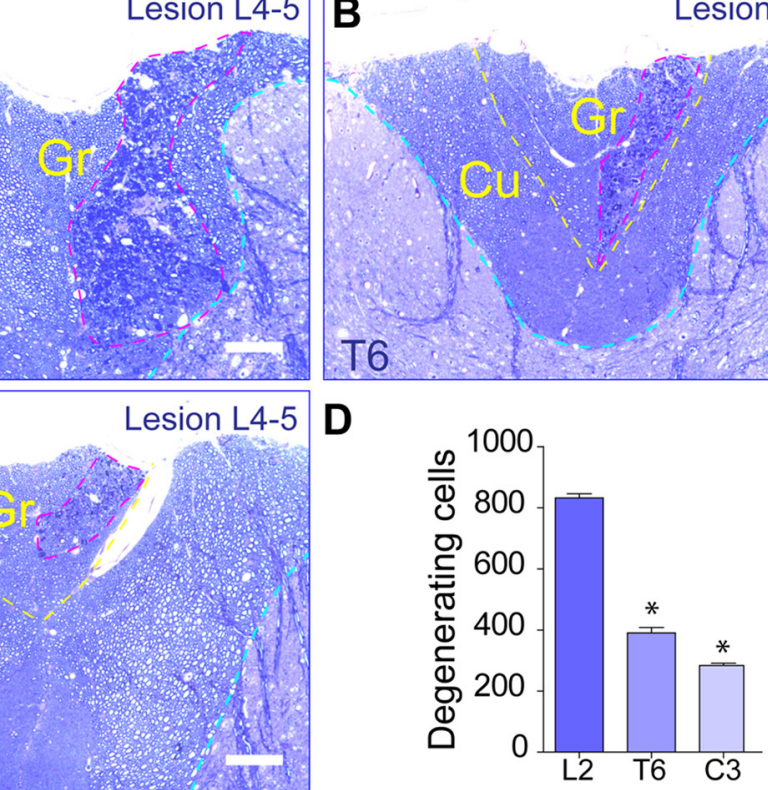

Figure 15. Central root rhizotomy suggests that lumbar proprioceptors drop out of the ascending pathway. $A-D$, Semithin 18) compared with L2 $(832 \pm 14) . n=3$ mice. ${ }^{*} p<0.001$. E, lllustration of the model that lumbar proprioceptors drop out the ascending pathway. Red represents RA mechanoreceptors; green represents proprioceptors. Dark color represents fibers from posterior DRGs, and lighter color represents fibers from anterior DRGs.

model because this modality-based segregation is observed in the caudal gracile fasciculus. Instead, afferents of most RA mechanoreceptors and proprioceptors must be sorted upon entering the dorsal column, as revealed by our single-cell analysis (Fig. 13). Interestingly, this modality-based organization is also obvious when we analyzed the distribution of small- and large-diameter axons in the dorsal column (Figs. 7 and 9), which coarsely correlate to mechanosensory and proprioceptive axons. Using this approach, we revealed that this modality-based functional organization is likely to be conserved across multiple mammalian species, including human (Figs. 10 and 11). Together, our results provide strong anatomical evidence that somatosensory afferents are organized by modality in the mammalian DDC pathway.

\section{A rough "somatotopic map" in the DDC pathway}

If the mammalian DDC pathway is primarily organized by modality, why has a "somatotopic map" been robustly observed? One of our observations using sparse genetic tracing may help to answer this question: ascending axons of RA mechanoreceptors enter the dorsal column 3-4 segments rostral than proprioceptors from the same DRG (Fig. 13C,D), which would generate a caudal/rostral somatotopic relationship between the two modalities (intermodality somatotopic organization) at any given spinal cord segment. Given the somatotopic organization existing within the same modality (Fig. 16A) (Whitsel et al., 1969, 1970), a rough "somatotopic map" is formed from medial to lateral 

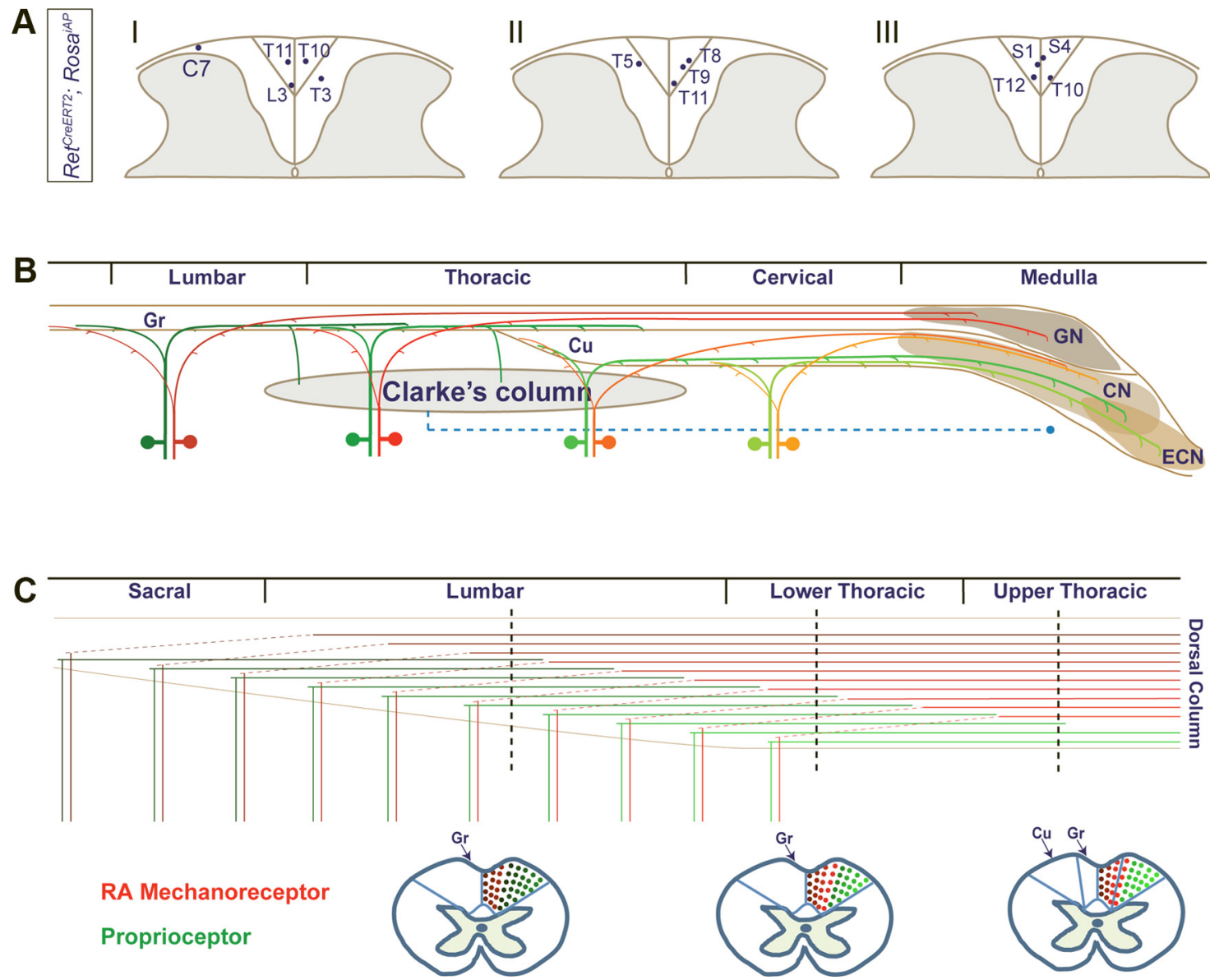

Figure 16. Model of the functional organization of the mammalian dorsal column. $A$, Relative positions of labeled ascending axons of RA mechanoreceptors from different DRGs are replotted as the transverse view at $\mathrm{C}$, suggesting that RA mechanosensory axons are organized in a somatotopic manner in the DDC pathway. $\boldsymbol{B}$, Coexisting model of the functional organization of the mammalian dorsal column. Schematic view of different morphologies of caudal and rostral RA mechanoreceptors and proprioceptors, which lead to modality segregation throughout the dorsal column and dorsal column nuclei. Caudal proprioceptors send collaterals to innervate the Clarke's column, which transmit the caudal proprioceptive information via the indirect pathway to the medulla. Most other collaterals of proprioceptors and RA mechanoreceptors are omitted for simplicity. C, Simulation to demonstrate how a modality-based organization can generate a rough somatotopic map in the caudal spinal cord dorsal column. Red lines indicate axons of RA mechanoreceptors; green lines indicate axons of proprioceptors; the dark to light gradient of color indicates caudal to rostral segments. In addition, dashed red lines indicate RA mechanosensory axons after bifurcation and before they enter the dorsal column. For this simulation, ascending axons of RA mechanoreceptors project $\sim 3$ segments rostrally on average before joining the dorsal column, whereas ascending axons of lumbar proprioceptors terminate after traveling $\sim 6.5$ segments. These numbers are derived from the experimental observations (Fig. 13J).

dorsal columns (Fig. 16C). Nevertheless, because the organizing mechanism is predominantly based on modality, this "somatotopic map" would usually break when the modality shifts. Indeed, supporting evidence can be seen from previous physiological recordings (Whitsel et al., 1969, 1970; Dykes, 1983). Thus, although the dorsal column and DCNs exhibit a rough "somatotopic map" at the phenotypic level, DDC somatosensory afferents are not simply organized based on their "receptive fields."

\section{Pre-target axon sorting in the DDC pathway}

Mechanoreceptors and proprioceptors tend to synapse with medulla neurons that receive the same type of inputs (modalityspecific convergence) (Dykes et al., 1982; Rasmusson and Northgrave, 1997). However, how this "modality-specific convergence" is established during development is unclear. Here we demonstrated that ascending axons of mechanoreceptors and proprioceptors are largely segregated from each other in the dorsal column before DCN innervation (the pretarget axon sorting), similar to primary olfactory axons (Imai et al., 2009). As a result, ascending axons of mechanoreceptors and proprioceptors innervate distinct domains of DCNs (Figs. 2 and 6), which would greatly facilitate the modality-specific convergence in the medulla. Thus, modality-based axon segregation in the DDC pathway could play an important role in establishing the correct mechanosensory and proprioceptive circuits.

\section{The indirect proprioceptive pathway}

Although previous anatomical and physiological studies suggested that some ascending proprioceptive axons from caudal DRGs terminate in the middle of the dorsal column (Burgess and Clark, 1969; Clark, 1972; Giuffrida and Rustioni, 1992), it is unclear whether this is true for all caudal proprioceptors or whether ascending axons of some mechanoreceptors also terminate. Here we examined ascending axons of hundreds of proprioceptors and RA mechanoreceptors using sparse genetic tracing. We found that ascending proprioceptive axons from $\mathrm{T} 6$ and above reach the medulla, whereas those below T6 travel a few segments and terminate in the dorsal column (Fig. 13J). In contrast, almost all RA mechanoreceptors project to the medulla, regardless of their soma location (Fig. 13I). Thus, our data provide the direct evidence that ascending axons of all caudal proprioceptors, but not mechanoreceptors, terminate in the middle of the dorsal column. 
The proprioceptive information from hindlimbs and the lower part of the body is therefore transmitted to brain via an indirect pathway, presumably through the Clarke's column (Matthews, 1982). Although the best known projection of the Clarke's column is the spinocerebellar tract (Mann, 1973), which relays caudal proprioceptive information to the cerebellum to coordinate movement, it also projects to the $\mathrm{Z}$ nucleus, $\mathrm{X}$ nucleus, and the rostral part of the CN in medulla (Landgren and Silfvenius, 1971, 1977), where relay neurons project to thalamus (Landgren and Silfvenius, 1971) to mediate proprioception.

\section{Coexistence of modality-based and somatotopic organization in the somatosensory system}

The somatosensory system displays both somatotopic and modality-based organizations, which encode the "where" and "what" information of a stimulus. However, how somatotopic and modality-based organizations coexist in the somatosensory system remains an intriguing question (Dykes, 1983). Our study suggests that the central somatosensory pathway is mainly organized by modality, with a somatotopic map in the same modality (a modality/somatotopy organization). Our results would predict that more than one somatotopic map is formed along the central pathway. Indeed, multiple homunculi have been identified in the thalamus (Dykes et al., 1981) and primary somatosensory cortex (Kaas et al., 1979). Intriguingly, peripheral somatosensory axons, which carry different modalities of somatosensory information but share similar receptive fields, usually fasciculate and project together. Thus, the peripheral somatosensory pathway seems to be organized in a somatotopy/ modality manner. How the central and peripheral axons of somatosensory neurons are organized by the opposite principles remains to be determined.

\section{Implication of the new model}

Dorsal column lesion is commonly found in spinal cord injury (Neumann and Woolf, 1999; Hollis and Zou, 2012) and diseases (Boshes and Padberg, 1953; Nathan et al., 1986). Thus, our results would have many implications for the dorsal column-related research and clinical practices. For example, some patients with dorsal column lesion show dissociated loss of vibration and proprioception (Ross, 1991), which could be readily explained by our model. In addition, our model suggests that, to regenerate the correct mechanosensory and proprioceptive circuits in the DCNs after spinal cord injury, it is necessary to promote differential growth of mechanosensory and proprioceptive axons and maintain their modality segregation. Last, dorsal column stimulation is effective for treating patients with brain injury (Oliveira and Fregni, 2011), neurodegenerative diseases (Fuentes et al., 2009), and chronic pain (Saulino and Shaw, 2012). However, pan-dorsal column stimulation has many side effects, such as paralysis, which might be caused by overexcitation of proprioceptors. Our results could potentially enable the development of focal and modality-specific dorsal column stimulation, which may reduce undesirable side effects.

\section{References}

Badea TC, Nathans J (2004) Quantitative analysis of neuronal morphologies in the mouse retina visualized by using a genetically directed reporter. J Comp Neurol 480:331-351. CrossRef Medline

Badea TC, Hua ZL, Smallwood PM, Williams J, Rotolo T, Ye X, Nathans J (2009) New mouse lines for the analysis of neuronal morphology using CreER(T)/loxP-directed sparse labeling. PLoS One 4:e7859. CrossRef Medline

Boshes B, Padberg F (1953) Studies on the cervical spinal cord of man: sensory pattern after interruption of the posterior columns. Neurology 3:90-101. CrossRef Medline

Brown AG, ed (1981) Organization in the spinal cord. New York: Springer.

Burgess PR, Clark FJ (1969) Characteristics of knee joint receptors in the cat. J Physiol 203:317-335. Medline

Clark FJ (1972) Central projection of sensory fibers from the cat knee joint. J Neurobiol 3:101-110. CrossRef Medline

Culberson JL, Brushart TM (1989) Somatotopy of digital nerve projections to the cuneate nucleus in the monkey. Somatosens Mot Res 6:319-330. CrossRef Medline

de Nooij JC, Doobar S, Jessell TM (2013) Etv1 inactivation reveals proprioceptor subclasses that reflect the level of NT3 expression in muscle targets. Neuron 77:1055-1068. CrossRef Medline

Dykes RW (1983) Parallel processing of somatosensory information: a theory. Brain Res 287:47-115. Medline

Dykes RW, Sur M, Merzenich MM, Kaas JH, Nelson RJ (1981) Regional segregation of neurons responding to quickly adapting, slowly adapting, deep and Pacinian receptors within thalamic ventroposterior lateral and ventroposterior inferior nuclei in the squirrel monkey (Saimiri sciureus). Neuroscience 6:1687-1692. CrossRef Medline

Dykes RW, Rasmusson DD, Sretavan D, Rehman NB (1982) Submodality segregation and receptive-field sequences in cuneate, gracile, and external cuneate nuclei of the cat. J Neurophysiol 47:389-416. Medline

Ernfors P, Lee KF, Kucera J, Jaenisch R (1994) Lack of neurotrophin-3 leads to deficiencies in the peripheral nervous system and loss of limb proprioceptive afferents. Cell 77:503-512. CrossRef Medline

Fleming MS, Ramos D, Han SB, Zhao J, Son YJ, Luo W (2012) The majority of dorsal spinal cord gastrin releasing peptide is synthesized locally whereas neuromedin B is highly expressed in pain- and itch-sensing somatosensory neurons. Mol Pain 8:52. CrossRef Medline

Fuentes R, Petersson P, Siesser WB, Caron MG, Nicolelis MA (2009) Spinal cord stimulation restores locomotion in animal models of Parkinson's disease. Science 323:1578-1582. CrossRef Medline

Fyffe RE, Cheema SS, Rustioni A (1986) Intracellular staining study of the feline cuneate nucleus: I. Terminal patterns of primary afferent fibers. J Neurophysiol 56:1268-1283. Medline

Giuffrida R, Rustioni A (1992) Dorsal root ganglion neurons projecting to the dorsal column nuclei of rats. J Comp Neurol 316:206-220. CrossRef Medline

Hippenmeyer S, Vrieseling E, Sigrist M, Portmann T, Laengle C, Ladle DR, Arber S (2005) A developmental switch in the response of DRG neurons to ETS transcription factor signaling. PLoS Biol 3:e159. CrossRef Medline

Hollis ER 2nd, Zou Y (2012) Reinduced Wnt signaling limits regenerative potential of sensory axons in the spinal cord following conditioning lesion. Proc Natl Acad Sci U S A 109:14663-14668. CrossRef Medline

Hummelsheim H, Wiesendanger R, Wiesendanger M, Bianchetti M (1985) The projection of low-threshold muscle afferents of the forelimb to the main and external cuneate nuclei of the monkey. Neuroscience 16:979 987. CrossRef Medline

Imai T, Yamazaki T, Kobayakawa R, Kobayakawa K, Abe T, Suzuki M, Sakano $\mathrm{H}$ (2009) Pre-target axon sorting establishes the neural map topography. Science 325:585-590. CrossRef Medline

Jackson SJ, Lee J, Nikodemova M, Fabry Z, Duncan ID (2009) Quantification of myelin and axon pathology during relapsing progressive experimental autoimmune encephalomyelitis in the Biozzi ABH mouse. J Neuropathol Exp Neurol 68:616-625. CrossRef Medline

Johansson H, Silfvenius H (1977) Connexions from large, ipsilateral hind limb muscle and skin afferents to the rostral main cuneate nucleus and to the nucleus X region in the cat. J Physiol 265:395-428. Medline

Johnson JI Jr, Welker WI, Pubols BH Jr (1968) Somatotopic organization of raccoon dorsal column nuclei. J Comp Neurol 132:1-43. CrossRef Medline

Kaas JH, Nelson RJ, Sur M, Lin CS, Merzenich MM (1979) Multiple representations of the body within the primary somatosensory cortex of primates. Science 204:521-523. CrossRef Medline

Landgren S, Silfvenius H (1971) Nucleus Z, the medullary relay in the projection path to the cerebral cortex of group I muscle afferents from the cat's hind limb. J Physiol 218:551-571. Medline

Li L, Rutlin M, Abraira VE, Cassidy C, Kus L, Gong S, Jankowski MP, Luo W, Heintz N, Koerber HR, Woodbury CJ, Ginty DD (2011) The functional organization of cutaneous low-threshold mechanosensory neurons. Cell 147:1615-1627. CrossRef Medline 
Ling LJ, Honda T, Shimada Y, Ozaki N, Shiraishi Y, Sugiura Y (2003) Central projection of unmyelinated (C) primary afferent fibers from gastrocnemius muscle in the guinea pig. J Comp Neurol 461:140-150. CrossRef Medline

Luo W, Wickramasinghe SR, Savitt JM, Griffin JW, Dawson TM, Ginty DD (2007) A hierarchical NGF signaling cascade controls Ret-dependent and Ret-independent events during development of nonpeptidergic DRG neurons. Neuron 54:739-754. CrossRef Medline

Luo W, Enomoto H, Rice FL, Milbrandt J, Ginty DD (2009) Molecular identification of rapidly adapting mechanoreceptors and their developmental dependence on ret signaling. Neuron 64:841-856. CrossRef Medline

Madisen L, Zwingman TA, Sunkin SM, Oh SW, Zariwala HA, Gu H, Ng LL, Palmiter RD, Hawrylycz MJ, Jones AR, Lein ES, Zeng H (2010) A robust and high-throughput Cre reporting and characterization system for the whole mouse brain. Nat Neurosci 13:133-140. CrossRef Medline

Mann MD (1973) Clarke's column and the dorsal spinocerebellar tract: a review. Brain Behav Evol 7:34-83. CrossRef Medline

Maslany S, Crockett DP, Egger MD (1991) Somatotopic organization of the dorsal column nuclei in the rat: transganglionic labelling with B-HRP and WGA-HRP. Brain Res 564:56-65. CrossRef Medline

Matthews PB (1982) Where does Sherrington's "muscular sense" originate? Muscles, joints, corollary discharges? Annu Rev Neurosci 5:189-218. CrossRef Medline

Molliver DC, Wright DE, Leitner ML, Parsadanian AS, Doster K, Wen D, Yan Q, Snider WD (1997) IB4-binding DRG neurons switch from NGF to GDNF dependence in early postnatal life. Neuron 19:849-861. CrossRef Medline

Nathan PW, Smith MC, Cook AW (1986) Sensory effects in man of lesions of the posterior columns and of some other afferent pathways. Brain 109:1003-1041. CrossRef Medline

Neumann S, WoolfCJ (1999) Regeneration of dorsal column fibers into and beyond the lesion site following adult spinal cord injury. Neuron 23:8391. CrossRef Medline

Nord SG (1967) Somatotopic organization in the spinal trigeminal nucleus, the dorsal column nuclei and related structures in the rat. J Comp Neurol 130:343-356. CrossRef Medline

Oliveira L, Fregni F (2011) Pharmacological and electrical stimulation in chronic disorders of consciousness: new insights and future directions. Brain Inj 25:315-327. CrossRef Medline

Rasmusson DD, Northgrave SA (1997) Reorganization of the raccoon cuneate nucleus after peripheral denervation. J Neurophysiol 78:29242936. Medline

Ross RT (1991) Dissociated loss of vibration, joint position and discriminatory tactile senses in disease of spinal cord and brain. Can J Neurol Sci 18:312-320. Medline

Saulino M, Shaw E (2012) New devices and drugs on the horizon in pain management. J Neurosurg Sci 56:313-322. Medline

Shi KSR, Motajo O, Kretschmer F, Mu X, Badea TC (2013) Genetic interactions between $\mathrm{Brn} 3$ transcription factors in retinal ganglion cell type specification. PLOS One 8:e76347. CrossRef

Smith MC, Deacon P (1984) Topographical anatomy of the posterior columns of the spinal cord in man: the long ascending fibres. Brain 107:671698. CrossRef Medline

Taniguchi H, He M, Wu P, Kim S, Paik R, Sugino K, Kvitsani D, Fu Y, Lu J, Lin Y, Miyoshi G, Shima Y, Fishell G, Nelson SB, Huang ZJ (2011) A resource of Cre driver lines for genetic targeting of GABAergic neurons in cerebral cortex. Neuron 71:995-1013. CrossRef Medline

Uddenberg N (1968) Differential localization in dorsal funiculus of fibres originating from different receptors. Exp Brain Res 4:367-376. Medline

Watson C, Kayalionglu G, eds (2009) The spinal cord. New York: Elsevier.

Whitsel BL, Petrucelli LM, Sapiro G (1969) Modality representation in the lumbar and cervical fasciculus gracilis of squirrel monkeys. Brain Res 15:67-78. CrossRef Medline

Whitsel BL, Petrucelli LM, Sapiro G, Ha H (1970) Fiber sorting in the fasciculus gracilis of squirrel monkeys. Exp Neurol 29:227-242. CrossRef Medline

Willis WD, ed (1991) Sensory mechanisms of the spinal cord, 2nd Ed. New York: Plenum. 